\title{
Boson localization and the superfluid-insulator transition
}

\author{
Matthew P. A. Fisher \\ IBM Research Division, Thomas J. Watson Research Center, Yorktown Heights, New York 10598 \\ Peter B. Weichman \\ Condensed Matter Physics 114-36, California Institute of Technology, Pasadena, California 91125 \\ G. Grinstein \\ IBM Research Division, Thomas J. Watson Research Center, Yorktown Heights, New York 10598 \\ Daniel S. Fisher \\ Joseph Henry Laboratory of Physics, Jadwin Hall, Princeton University, Princeton, New Jersey 08544
}

(Received 15 November 1988)

\begin{abstract}
The phase diagrams and phase transitions of bosons with short-ranged repulsive interactions moving in periodic and/or random external potentials at zero temperature are investigated with emphasis on the superfluid-insulator transition induced by varying a parameter such as the density. Bosons in periodic potentials (e.g., on a lattice) at $T=0$ exhibit two types of phases: a superfluid phase and Mott insulating phases characterized by integer (or commensurate) boson densities, by the existence of a gap for particle-hole excitations, and by zero compressibility. Generically, the superfluid onset transition in $d$ dimensions from a Mott insulator to superfluidity is "ideal," or mean field in character, but at special multicritical points with particle-hole symmetry it is in the universality class of the $(d+1)$-dimensional $X Y$ model. In the presence of disorder, a third, "Bose glass" phase exists. This phase is insulating because of the localization effects of the randomness and analogous to the Fermi glass phase of interacting fermions in a strongly disordered potential. The Bose glass phase is characterized by a finite compressibility, no gap, but an infinite superfluid susceptibility. In the presence of disorder the transition to superfluidity is argued to occur only from the Bose glass phase, and never directly from the Mott insulator. This zero-temperature superfluidinsulator transition is studied via generalizations of the Josephson scaling relation for the superfluid density at the ordinary $\lambda$ transition, highlighting the crucial role of quantum fluctuations. The transition is found to have a dynamic critical exponent $z$ exactly equal to $d$ and correlation length and order-parameter correlation exponents $v$ and $\eta$ which satisfy the bounds $v \geq 2 / d$ and $\eta \leq 2-d$, respectively. It is argued that the superfluid-insulator transition in the presence of disorder may have an upper critical dimension $d_{c}$ which is infinite, but a perturbative renormalization-group calculation wherein the critical exponents have mean-field values for weak disorder above $d=4$ is also discussed. Many of these conclusions are verified by explicit calculations on a model of onedimensional bosons in the presence of both random and periodic potentials. The general results are applied to experiments on ${ }^{4} \mathrm{He}$ absorbed in porous media such as Vycor. Some measurable properties of the superfluid onset are predicted exactly [e.g., the exponent $x$ relating the $\lambda$ transition temperature to the zero-temperature superfluid density is found to be $d / 2(d-1)$ ], while stringent bounds are placed on others. Analysis of preliminary data is consistent with these predictions.
\end{abstract}

\section{INTRODUCTION}

During the past dozen years, a great deal of attention has been lavished on the problem of metal-insulator transitions in Fermi systems. ${ }^{1}$ Yet, in spite of this, the understanding of these phenomena is still rather fragmentary. Perhaps surprisingly, far less attention has been paid to the analogous problem for bosons: the transition at zero temperature from an insulating to a conducting phase. This is true in spite of the natural experimental realizations of ${ }^{4} \mathrm{He}$ absorbed in porous media or on various substrates, and granular superconductors in which the Cooper pairs may act, at least approximately, like bosons.

For pure Bose systems, the conducting phase at zero temperature is presumably always superfluid ${ }^{2}$ so that the conducting-insulator transition corresponds to the onset of superfluidity. In contrast to Fermi systems, there is thus a natural order parameter for the Bose problem, associated with the off-diagonal long-range order of the superfluid phase. In principle, this should allow the onset of superfluidity at zero temperature to be treated by similar techniques to those for conventional phase transitions, rather than the less well-understood-and more directly perturbative - techniques used for metal-insulator transitions.

For bosons in a random potential, repulsive interactions are essential in order to stop all the particles condensing into the lowest localized eigenstate. Thus, in contrast to Fermi systems, there is no sensible noninteracting starting point about which to perturb. Indeed, the onset of superfluidity at zero temperature is a conse- 
quence of the competition between the kinetic energy, which tries to delocalize the particles and reduce the phase fluctuations of the Bose field, and the combination of the interactions and the random potential which try to localize the particles and make the number density fluctuations small. This competition plays an essential role in the scaling analysis of the superfluid onset transition which was briefly introduced in Ref. 3 and is discussed in more detail here.

In this paper we discuss the behavior of bosons with short-range repulsive interactions moving in both random and periodic external potentials. We argue that, in general, there can be three types of phases at zero temperature: a superfluid phase, commensurate Mott insulating phases in which there is a gap for particle-hole excitations and zero compressibility, and a "Bose glass" phase in which there is no gap, the compressibility is finite, but the system is an insulator because of the localization effects of the random potential. This Bose glass phase, which is rather analogous to the Fermi glass phase of interacting fermions in a strongly disordered potential, with the repulsive interactions playing the role of Pauli exclusion, has some rather surprising properties, particularly an infinite superfluid susceptibility. The principal focus of this paper is the onset of superfluidity at zero temperature as the parameters of the system are varied.

Two groups have recently studied the onset of superfluidity in a random potential. Ma, Halperin, and Lee $(\mathrm{MHL})^{4}$ have attempted a Landau theory and dimensionality expansion about a mean-field theory; we believe (and will argue) that this work contains an error which invalidates the conclusions. Giamarchi and Schulz, ${ }^{5}$ on the other hand, have analyzed the interacting Bose problem in one dimension by a renormalization-group calculation perturbation in the strength of the disorder. We will rely heavily on this calculation as a cornerstone on which to test more general scaling arguments.

We will argue that, in contrast to natural expectations, the onset of superfluidity at zero temperature is generally not in the universality class of the $d+1$-dimensional $X Y$ model (with, in the presence of randomness, a random time-independent potential). Instead, we will show that in the absence of randomness, such $d+1$-dimensional $X Y$ models describe only special multicritical transitions while generically the behavior is that of a zero-density transition such as that which occurs as the density of bosons is increased from zero in the absence of an external potential. $^{6-8}$ (This is also the case for the generic quantum $X Y$ magnet without time reversal invariance.) In the presence of randomness, we expect the transition to superfluidity always to occur from the Bose glass phase. This transition, as argued in Ref. 3, is characterized by a dynamic critical exponent $z$ which because of numberphase competition turns out to be equal to the spatial dimension $d$, a correlation length exponent $v \geq 2 / d$, and an order-parameter exponent $\eta$. This latter exponent is argued here to satisfy the bound $\eta \leq 2-d$. These exponent relations, when placed in the framework of a scaling theory, enable explicit and verifiable predictions for various static and dynamic properties near the zerotemperature superfluid onset transition. Some measur- able exponents, depending only on $z$, are predicted exactly.

We present arguments that there may, in fact, be no simple high-dimensional limit of this transition - at least not of a conventional Gaussian or mean-field kind-and that the equality $z=d$ holds in all dimensions. We also outline an alternate possibility, discussed by Weichman and Kim in Ref. 8, that for $d>4$ there are two universality classes, one for strong disorder with presumably $z=d$ and the other for weak disorder with mean-field exponents.

The outline of this paper is as follows: In Sec. II the basic model of bosons hopping on a lattice is introduced. Its relation to the usual charging models of granular superconductivity ${ }^{9,10}$ is briefly explained. By treating the kinetic energy (i.e., hopping) as a perturbation, the phase diagram in the hopping strength, $J$, and chemical potential, $\mu$, plane is worked out. For the pure, nonrandom, system we find two types of phases: a set of incompressible Mott insulating phases in which the density is fixed commensurately at a positive integer, $n$, per site; and a superfluid phase with the usual off-diagonal long-range order (Fig. 1). In the random case we argue that a gapless, insulating Bose glass phase with nonzero compressibility, must intervene between the Mott and superfluid phases (Fig. 2), and that, in fact, the Mott phase can be destroyed completely if the randomness is sufficiently strong (this is almost certainly the relevant case for the phase diagram of ${ }^{4} \mathrm{He}$ adsorbed in porous media). In Appendix $A$ we derive the exact phase diagrams within a mean-field theory (i.e., an infinite-range hopping model), verifying many of the general details, but finding no Bose glass phase. This, however, is hardly surprising since localization effects are absent when hopping can occur between any two sites, particularly those with degenerate onsite energy.

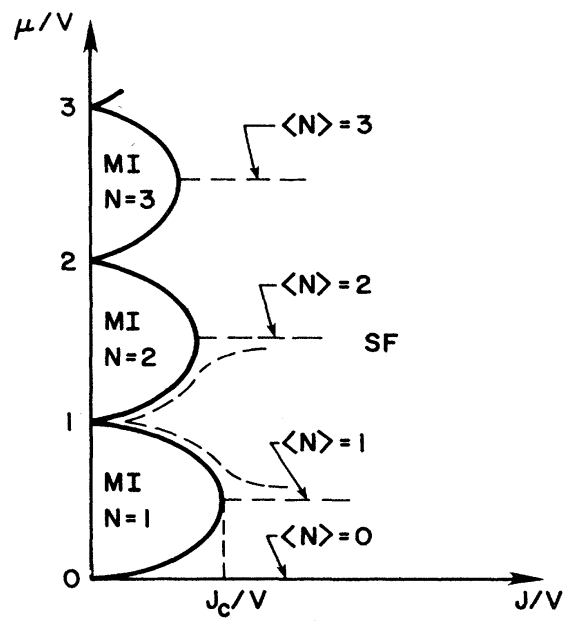

FIG. 1. Zero-temperature phase diagram for the lattice model of interacting bosons, (2.1), in the absence of disorder. For an integer number of bosons per site the superfluid phase (SF) is unstable to a Mott insulating (MI) phase at small $J / V$. 
In Sec. III we expand upon a scaling theory, outlined in Ref. 3, for the superfluid transition in the presence of disorder. Following Ma, Halperin, and Lee, ${ }^{4}$ we generalize the Josephson relation, relating the superfluid density
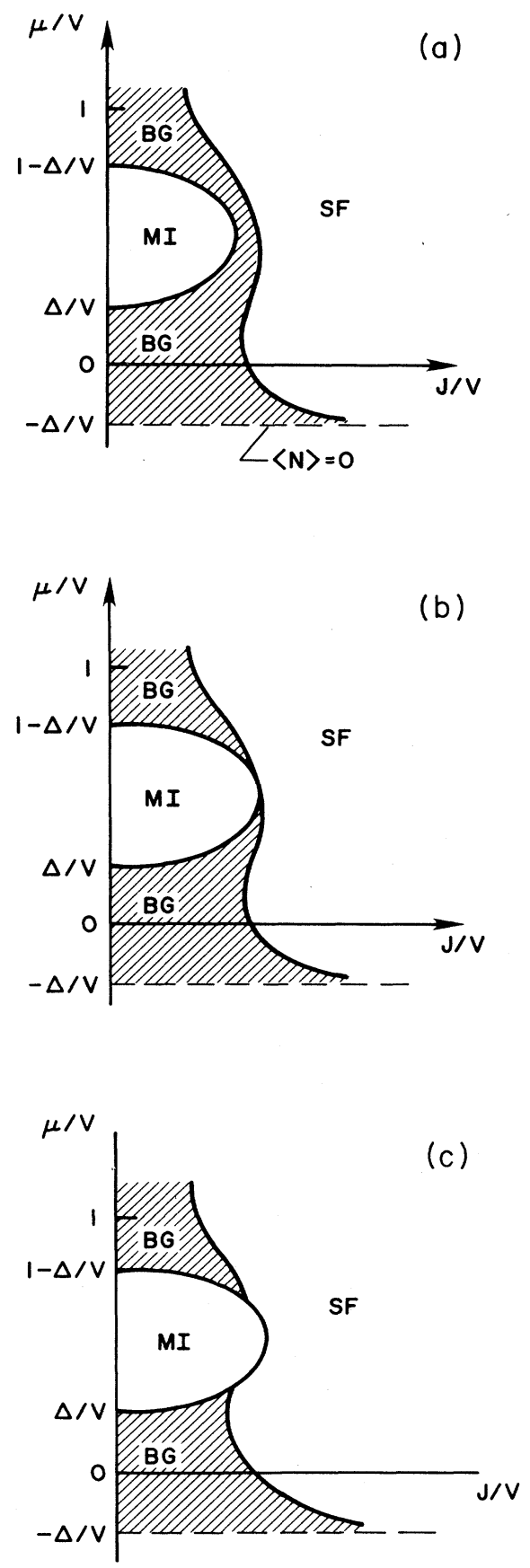

FIG. 2. Possible zero-temperature phase diagrams for the lattice boson model (2.1) with weak bounded disorder, $\Delta / V<\frac{1}{2}$. Figure 2(a), where the transition to superfluidity occurs only from the insulating, gapless Bose glass phase (BG), is argued in the text to be the correct phase diagram. critical exponent to the correlation length exponent at the $\lambda$ transition, ${ }^{11}$ to the zero-temperature superfluid onset transition. In contrast to the $\lambda$ transition, the zerotemperature onset transition is driven entirely by quantum fluctuations, so that the static and dynamics are inextricably linked. As a consequence, the dynamical exponent $z$ enters into the generalized Josephson relation for the zero-temperature transition. Using the existence of a well-defined superfluid hydrodynamical action, describing long-wavelength and low-energy phase fluctuations of the superfluid order parameter, as well as a bound on the correlation length exponent, ${ }^{12}$ we argue that one must have the equality $z=d$. Moreover, by considering the single-particle density of states near criticality, the order-parameter exponent $\eta$ is argued to be bounded above by $2-d$.

In Sec. IV we outline and extend what is known about microscopic calculations of the critical phenomena of superfluid onset at zero temperature. For the continuum Bose gas with no disorder, this regime is described completely by the Bogoliubov model. ${ }^{8}$ The continuum Bose gas results also hold for the pure lattice Bose gas, except for a special set of multicritical points on the Mottsuperfluid phase boundary. The special multicritical points correspond to the maxima of the lobes in Fig. 1, where the transition takes place at fixed commensurate (integer) density. There the behavior is that of the classical $X Y$ model in $(d+1)$ dimensions. ${ }^{9,10}$

The more substantial results in Sec. IV involve the treatment of bosons in one dimension, in the presence of both random and periodic external potentials. Many of the results can be transcribed directly from the work of Giamarchi and Schultz. ${ }^{5}$ The calculations are based on a representation of the Bose Hamiltonian first introduced by Haldane, ${ }^{13}$ which can also be generalized to higher dimensions. ${ }^{14}$ We briefly review this approach in Appendix B. The superfluid transition in a purely periodic potential can basically be interpreted as a $2 d$ KosterlitzThouless roughening transition, the smooth phase corresponding to the insulating Mott phase. In the presence of randomness, sufficiently weak periodic potentials are in fact irrelevant, and a unique superfluid onset is found with exponents which confirm those deduced from scaling arguments in Sec. III. The universality class is not Kosterlitz-Thouless, as would have been expected if the model were simply $(d+1)-X Y$, as assumed by MHL. ${ }^{4}$

In the last part of Sec. IV, attempts to find an $\varepsilon$ expansion, or generalization thereof, about $d=4$ are outlined, and the difficulties encountered are summarized.

In Sec. $\mathrm{V}$ we present some experimental ramifications appropriate to systems of ${ }^{4} \mathrm{He}$ absorbed in porous media, ${ }^{15,16}$ at low temperatures and at densities close to the critical density $\rho_{c}$ of the $T=0$ superfluid onset transition. By applying the relationship $z=d$, obtained in Sec. III for the Bose glass to superfluid transition, and the general bound on the correlation length exponent, $v \geq 2 / d$, stringent bounds can be placed on various experimentally accessible exponents. More surprisingly, some exponents, depending only on $z$ (and $d$ ) can be predicted exactly. Specifically, we predict that the exponent $x$, which relates [via $\left.T_{c} \sim \rho_{s}^{x}(0)\right]$ the zero-temperature 
superfluid density, $\rho_{s}(0)$, to the $\lambda$-transition temperature $T_{c}$ as the overall density is decreased toward $\rho_{c}$, satisfies $x=d / 2(d-1)$ for all $d \geq 2$. Analysis of some very preliminary data ${ }^{17}$ yield exponent values consistent with these theoretical predictions.

Finally, in the last part of Sec. V we briefly summarize our main conclusions.

\section{PHASE DIAGRAMS FOR THE BOSE LATTICE GAS}

\section{A. Models}

In this section we construct zero-temperature $(T=0)$ phase diagrams for models consisting of soft-core bosons hopping on a lattice, both with and without disorder. The Hamiltonian of interest takes the form $\hat{\mathbf{H}}=\widehat{\mathbf{H}}_{0}+\widehat{\mathbf{H}}_{1}$ with

$$
\begin{aligned}
& \hat{\mathbf{H}}_{0}=-\sum_{i}\left(-J_{0}+\mu+\delta \mu_{i}\right) \hat{\mathbf{N}}_{i}+\frac{1}{2} V \sum_{i} \widehat{\mathbf{N}}_{i}\left(\widehat{\mathbf{N}}_{i}-1\right), \\
& \hat{\mathbf{H}}_{1}=-\frac{1}{2} \sum_{i, j} J_{i j}\left(\hat{\boldsymbol{\Phi}}_{i}^{+} \boldsymbol{\Phi}_{j}+\text { H.c. }\right)
\end{aligned}
$$

where $\hat{\mathbf{N}}_{i}=\hat{\boldsymbol{\Phi}}_{i}^{+} \hat{\boldsymbol{\Phi}}_{i}$ and $\hat{\boldsymbol{\Phi}}_{i}$ is a boson field operator (on site $i$ ) which satisfies the standard commutation relations, $\left[\hat{\boldsymbol{\Phi}}_{i}, \hat{\boldsymbol{\Phi}}_{j}^{+}\right]=\delta_{i j}$. Here $J_{i j}$ is the strength of the hopping between sites $i$ and $j$ and $J_{0}=\Sigma_{j} J_{i j}$. The average chemical potential $\mu$ fixes the boson density, whereas $\delta \mu_{i}$ is a random on-site potential with zero mean. The on-site soft-core repulsion has strength $V$. For concreteness we consider two different distributions for the random potential, $\delta \mu_{i}$ :

(a) $\delta \mu_{i}$ uniformly distributed between $-\Delta$ and $\Delta$;

(b) an unbounded Gaussian distribution, $P\left(\delta \mu_{i}\right)=\exp \left(-\delta \mu_{i}^{2} / 2 \widetilde{\Delta}\right) /(2 \pi \widetilde{\Delta})^{1 / 2}$.

In either case, the random variables on different sites are taken to be independent.

The Hamiltonian (2.1) should be contrasted with several closely related but more extensively studied lattice boson models. In the hard-core limit $(V \rightarrow \infty)$, boson occupancy is restricted to zero or one, and the Hamiltonian can be expressed in terms of a spin- $\frac{1}{2}$ quantum $X X Z$ model. ${ }^{18}$ At $T=0$, and in the absence of disorder $(\Delta=0)$, such a model always describes a superfluid state, except in the (somewhat artificial) limit where there is exactly one boson per site. As we shall see, the soft-core boson model (2.1) exhibits a richer phase diagram, with, for example, a nontrivial normal-to-superfluid transition at $T=\Delta=0$ for fixed-integer Bose density.

Josephson junction array Hamiltonians of the form ${ }^{9,10}$

$$
\hat{\mathbf{H}}=\frac{1}{2} V \sum_{i} \hat{\mathbf{n}}_{i}^{2}-\sum_{i, j} J_{i j} \cos \left(\hat{\boldsymbol{\phi}}_{i}-\widehat{\boldsymbol{\phi}}_{j}\right)
$$

constitute another frequently studied lattice boson model. Here the phase operator $\widehat{\boldsymbol{\phi}}_{i}$ is canonically conjugate to $\widehat{\mathbf{n}}_{i}$, which measures the deviation of the boson density from the mean. As noted in Ref. 10, the model (2.3) can be obtained as a special case of $(2.1)$ by setting $\Delta=0$, choosing $\mu$ to fix the boson density at an integer per site, and then expressing the complex field $\Phi_{i}$ which appears in the path-integral representation of (2.1) in terms of an amplitude and phase $\left[\Phi_{i} \equiv\left|\Phi_{i}\right| \exp \left(i \phi_{i}\right)\right]$. Upon integrating out the (small) amplitude fluctuations to quadratic order, one arrives at a phase-only model which is identical to the path-integral representation of (2.3). However, since the eigenvalues of $\hat{\mathbf{n}}_{i}$ run from $-\infty$ to $\infty,(2.3)$ can be compared quantitatively to (2.1) only when the mean density, $N$, about which the $\widehat{\mathbf{n}}_{i}$ fluctuate, is large. Note that the $J_{i j}$ in (2.3) are really $N$ times the $J_{i j}$ in (2.1).

\section{B. Zero-temperature phase diagram for the pure system}

In this section we construct the zero-temperature phase diagram for the pure system (2.1), with $\Delta=0$ and $J_{i j}$ taken to represent uniform short-range hopping. For specificity we consider nearest-neighbor hopping of strength $J$, and study the phase diagram in the $\mu-J$ plane (Fig. 1). We begin with the trivial limit $J=0$, where each site $i$ is clearly occupied by the non-negative integer number $n$ of bosons which minimizes the on-site energy

$$
\varepsilon(n)=-\mu n+\frac{1}{2} V n(n-1) .
$$

Thus (Fig. 1) for all values of $\mu$ in the interval $n-1<\mu / V<n$ (where $n \geq 1$ ), exactly $n$ bosons occupy each site. For $\mu<0, n=0$.

Now imagine fixing $\mu$ at a value corresponding to $n$ bosons per site, i.e., $\mu / V=n-\frac{1}{2}+\alpha$, for some $\alpha$ in the range $-\frac{1}{2}<\alpha<\frac{1}{2}$, and turn on some weak hopping: $J>0$. Suppose $J$ is small compared to the lesser of the two on-site energies, $\delta E_{p} \sim\left(\frac{1}{2}-\alpha\right) V$ and $\delta E_{h} \sim\left(\frac{1}{2}+\alpha\right) V$, respectively, required to add or remove one particle from the system. Then the kinetic energy $(\sim J)$ gained by adding (removing) a particle from the system and allowing the extra particle (hole) to hop around the lattice is insufficient to overcome the potential energy cost. We conclude that for every positive $n$ there exists a finite region in the $\mu-J$ plane (Fig. 1) in which the number of particles is fixed at precisely $n$ per site. In each such region, moreover, allowing a boson to hop from one site to the next gains roughly $J$ in kinetic energy at the expense of $\delta E_{p h} \equiv \delta E_{p}+\delta E_{h}$ in potential energy. Since $J<\delta E_{p h}$, such hops are energetically costly. It follows readily (e.g., from trying to compute the ground-state wave function perturbatively in $J),{ }^{19}$ that the probability of a boson having hopped $r$ sites from its initial position is roughly proportional to $\left(J / \delta E_{p h}\right)^{r}$, or $\exp (-r / \xi)$, where 
$\xi \sim\left[\ln \left(\delta E_{p h} / J\right)\right]^{-1}$. Thus, the regions of fixed $n$ in Fig. 1 represent normal, insulating states wherein the density fluctuations are localized in a volume of linear size $\xi$. Since the constancy of $n$ implies that the compressibility, $\partial \rho / \partial \mu$, vanishes everywhere within these states, they are also incompressible; they are, in other words, Mott insulating phases.

The Mott insulating phases are characterized by the existence of an energy gap, $E_{g}$, for the creation of particle or hole excitations, i.e., for the addition of particles to, or removal of particles from, the system. For any point within a Mott insulating phase in Fig. $1, E_{g}$ for particle (hole) excitations is simply the distance in the $\mu$ direction, with $J$ fixed, from the upper (lower) phase boundary, i.e., the minimum distance which allows extra particles (holes) to be added. For small nonzero temperature at constant $\mu$, the mobility (or conductivity) of the Mott states has an activated form, $\exp \left(-\widetilde{E}_{g} / k_{B} T\right)$, where $\widetilde{E}_{g}$ is the smaller of the particle and hole gaps.

It should be emphasized that in each Mott insulator phase, the lowest-lying excitation which conserves total particle number is a particle-plus-hole excitation. The energy of this excitation is simply the sum of the particle and hole energies (i.e., equal to the difference in $\mu$ between the top and bottom phase boundary at given $J$ in Fig. 1) and is thus independent of $\mu$, depending only on $J$ within a given Mott phase. When the temperature is raised at fixed-integer density, it is one-half of this particle-plus-hole energy which occurs in the exponential characterizing the thermally activated mobility.

The fact that the Mott insulating phases have lobelike shapes corresponding roughly to those shown in Fig. 1 can be understood as follows: If one starts at a point of the $\mu$ - $J$ plane in one of the Mott insulating states and increases $\mu$ at fixed $J$, one will eventually reach a point where the kinetic energy gained by adding an extra particle and letting it hop around the system will balance the associated potential-energy cost. Since any nonzero density of particles free to hop without energy cost through the system will, at zero temperature, immediately Bose condense producing a superfluid state, it follows that this point of energy balance defines the phase boundary for a transition between the Mott insulating and superfluid phases. Similarly, decreasing $\mu$ from a point within one of the Mott insulating phases eventually makes it energetically favorable to remove bosons (create holes) in the system. The holes, being free to hop throughout the lattice, likewise condense into a superfluid state. Since the kinetic energy of mobile bosons or holes increases with $J$, the width in $\mu$ of the Mott state decreases with increasing $J$, producing the schematic phase diagram of Fig. 1. Note that the superfluid phase extends all the way down to $J=0$ at integer values of $\mu / V$, since $\mu / V=n$ implies that occupying a site with $n-1$ particles at $J=0$ is energetically identical to occupying it with $n$. Thus there is no energy barrier to the addition of extra particles, and superfluidity occurs at arbitrarily small $J$.

It is instructive to consider the contours of constant density in the phase diagram; the lines of integer density, $\left\langle\hat{\mathbf{N}}_{i}\right\rangle=n$, drawn schematically in Fig. 1, are of particular interest. Each such contour (which represents the canon- ical ensemble of the system at integer particle density $n$ ), meets the phase boundary of the corresponding Mott insulating state at the point of maximum $J$ on that phase boundary, as in Fig. 1. It is easy to see that were this not the case, i.e., if the line $\left\langle\hat{\mathbf{N}}_{i}\right\rangle=n$ in the superfluid state joined the corresponding Mott insulating lobe at a point other than its tip, then the compressibility would be negative in the vicinity of the tip, a physical impossibility. For (noninteger) densities between $n$ and $n+1$ say, the constant density contours lie entirely within the superfluid phase, skirting the Mott insulating phases (as in Fig. 1) and terminating on the $\mu$ axis at the special point $\mu / V=n$. This reflects our earlier argument that if some sites are occupied with $n$ bosons and some with $(n+1)$, the extra particles can hop around the lattice without energy cost, and so can Bose condense for arbitrarily small $J$.

For densities just slightly greater (less) than $n$, the constant-density contours lie just slightly above (below) the Mott insulating phase with $\left\langle\hat{\mathbf{N}}_{i}\right\rangle=n$. This is consistent with our previous assertion that the Mott insulating-superfluid transition at a generic point on the phase boundary is driven by the addition or subtraction of small numbers of particles to the incompressible Mott insulating phase. That is, the density changes continuously from its fixed-integer value, $n$, in the Mott insulating state as one crosses into the superfluid. The transition at fixed integer density, $n$, at the tip of a Mott insulating lobe, on the other hand, is driven by quite different physics: Here the density never changes, but sufficiently large $J$ enables the bosons to overcome the on-site repulsion and hop throughout the lattice anyhow, thereby Bose condensing into the superfluid state. One might suspect, therefore, that the fixed-density Mott insulatingsuperfluid transition at the tip of a lobe is in a different universality class from the generic, density-driven transition, i.e., is a special, multicritical transition. This suspicion [which will be verified in Sec. IV A, where we show that the transitions are $(d+1)$-dimensional $X Y$-like and mean-fieldlike, respectively] is supported by simple inspection of Fig. 1: It is clear that as one moves toward the tip of one of the Mott insulating lobes by increasing $J$ at fixed $\mu$, the particle (or hole) gap [defined above as the distance in $\mu$ to the upper (lower) phase boundary], vanishes as $E_{g} \sim\left(J_{c}-J\right)^{z v}$, with an exponent $z v$ which we later show is less than unity, as represented in Fig. 1. (Here $J_{c}$ is the value of $J$ right at the tip, and the rationale for denoting the exponent $z v$ will be explained in Sec. IV.) Approaching any other point on the phase boundary from the Mott insulating side by increasing $J$, at fixed $\mu$, however, clearly yields an exponent $z v$ of exactly 1 . This confirms the special character of the transition at fixed-integer density.

Most of the features of the phase diagram discussed above can be verified by explicit calculation on a meanfield model with infinite-range hopping: $J_{i j}=J / N$ for any two sites $i$ and $j$ in (2.1), where $N$ is the total number of sites. The exact solution of this model, whose phase diagram has precisely the topology of Fig. 1, is outlined in the Appendix. This solution reveals, moreover, that $J_{c}(n)$, the value of $J$ at the tip of the $n$th Mott lobe, varies 
as $J_{c}(n) \sim 1 / n$ for large $n$, and that in this mean-field limit $z v=\frac{1}{2}$ at the multicritical points, so that the phase boundary is parabolic.

\section{Zero-temperature phase diagram for the disordered system}

We now study the effect of disorder on the zerotemperature phase diagram deduced above. For concreteness we restrict attention to on-site randomness with uniform nearest-neighbor hopping of strength $J$. We begin with bounded randomness, case (2.2a), treating first the trivial limit where $J=0$. Then, each site $i$ contains the non-negative integer number, $n_{i}$ of bosons which minimizes the on-site energy,

$$
\varepsilon\left(n_{i}\right)=-\left(\mu+\delta \mu_{i}\right) n_{i}+\frac{1}{2} V n_{i}\left(n_{i}-1\right) .
$$

It is simply verified that for sufficiently weak disorder, viz., $\Delta<V / 2$, the $\mu$ axis breaks up into intervals of width $V-2 \Delta$, centered about the values $\left(n-\frac{1}{2}\right) V$, for $n=1,2,3, \ldots$. For any $\mu$ in the $n$th such interval, there are precisely $n$ bosons on each site, so that $\left\langle\hat{\mathbf{N}}_{i}\right\rangle=n$. Thus these intervals are precise analogs of the intervals on the $\mu$ axis in Fig. 1 for the pure system. The effect of the randomness is to produce gaps of width $2 \Delta$ between these intervals (Fig. 2). For values of $\mu$ in the gap between the $n$th and $(n+1)$ st intervals, i.e., for $n V-\Delta<\mu<n V+\Delta$, the occupation, $n_{i}$, of the $i$ th site is either $n$ or $n+1$, according as $\delta \mu_{i}$ is less or greater than $V n-\mu$; the average occupation, $\left\langle\hat{\mathbf{N}}_{i}\right\rangle$, thus increases linearly from $n$ to $n+1$ as $\mu$ increases from $n V-\Delta$ to $n V+\Delta$. Note that for $\mu<-\Delta / V,\left\langle\hat{\mathbf{N}}_{i}\right\rangle$ is strictly zero.

To study the effect of taking $J>0$, first consider values of $\mu$ in the interval between $(n-1) V+\Delta$ and $n V-\Delta$, where at $J=0$ there are exactly $n$ bosons per site. Suppose $J$ is positive but small. As in the pure case, values of $J \ll V$ are insufficient to overcome the repulsive on-site potential and allow extra particles to be added to occupied sites. Thus for every integer $n \geq 0$, there is a region in the $\mu-J$ phase wherein $\left\langle\hat{\mathbf{N}}_{i}\right\rangle$ is fixed at $n$; the integer intervals on the $\mu$ axis form the left boundaries [Fig. 2(a)] of these integer-density regions, which clearly represent incompressible Mott insulating states. All this is qualitatively as in the pure system. The main qualitative effect of the disorder is to produce a new, insulating, "Bose glass" state in the phase diagram.

To see this, consider a value of $\mu$ in the gap between the Mott states with $\left\langle\hat{\mathbf{N}}_{i}\right\rangle=n$ and $\left\langle\hat{\mathbf{N}}_{i}\right\rangle=n+1$ in Fig. 2(a). In the decoupled $(J=0)$ limit, sites $i$ with $\delta \mu_{i}$ less than or greater than $n V-\mu$ contain $n$ or $n+1$ bosons, respectively. When $J$ is made slightly positive, bosons can hop out of this $J=0$ configuration to nearest-neighbor sites, thereby gaining kinetic energy. In this region, perturbative arguments can be made which are similar to those for the strongly localized regime of noninteracting fermions. The interacting Bose system is, of course, more complicated but qualitatively the repulsive interactions play a role analogous to the Pauli exclusion between the fermions. One may attempt a perturbative expansion of the single-particle Green's function ${ }^{19}$ in powers of $J$, about the fully localized limit $J=0$. Most of the energy denominators associated with hops of a boson will be of order $V$ because of the interactions. Thus naively, the Green's function between sites a distance $r_{i j}$ apart will decay as $(J / V)^{r_{i j}}$, i.e., exponentially. Important subtleties will occur, however, because of resonances between pairs of sites on which the local potentials are close to each other: $\delta \mu_{i}-\delta \mu_{j} \leq(J / V)^{r_{i j}} J$. It is natural to expect that such resonances could be handled by similar techniques to those for conventional noninteracting localization. ${ }^{20}$ In particular, we expect that on large length scales, $L$, almost all regions of the system of linear size $L$ will be free of resonances implying that the Green's function decays exponentially except, perhaps, in the rare badly behaved regions. On scale $L$, one then has a renormalized Bose glass problem in which the effective disorder is stronger than in the original problem and the chance of resonances occurring at scale $2 L$ is smaller. Thus we expect that in the infinite system the Green's function will decay exponentially with probability one with a localization length $\xi \sim(\ln V / J)^{-1}$. This will also be the decay length for the superfluid correlations at zero temperature, since these are just given by the decay of the equal time singleparticle Green's function $\left\langle\Phi(r) \Phi^{+}\left(r^{\prime}\right)\right\rangle$.

In the well-localized regime of the Bose glass phase, the low-energy excitations are essentially quasiparticle or quasihole excitations $^{21}$ localized in regions of size $\xi$. In the absence of hopping, the density of states of these excitations is constant down to zero excitation energy, $\varepsilon$, because of the continuous distribution of the $\delta \mu_{i}$. We expect, from the above perturbative arguments, that this behavior persists in the presence of a small amount of hopping. Indeed, the density of states of the quasiparticle excitations will presumably be constant at zero energy throughout the entire Bose glass phase. ${ }^{21}$ Moreover, because the quasiparticles are localized in this phase, the single-particle density of states, ${ }^{19}$

$$
\rho_{1}(\omega) \equiv-\frac{1}{\pi V} \int d r \operatorname{Im} G(r, r ; \omega)
$$

will also be constant at zero energy $(\omega=0)$. [By contrast, in the Mott phase, $\rho_{1}(\omega)=0$ for $-E_{h}<\omega<E_{p}$ where $E_{p}$ and $E_{h}$ are the particle and hole gaps, respectively.]

A constant density of states in the Bose glass phase has bizarre consequences for the superfluid susceptibility. To see this, note that the ensemble averaged imaginary time Green's function,

$$
\overline{G(r, \tau)} \equiv \overline{\left\langle T_{\tau} \Phi(r, \tau) \Phi^{+}(0,0)\right\rangle},
$$

at equal positions $(r=0)$ can be related directly to the density of states,

$$
\overline{G(r=0, \tau)}=\int_{0}^{\infty} d \varepsilon e^{-\varepsilon|\tau|} \rho_{1}( \pm \varepsilon),
$$

where the $+(-)$ sign is for $\tau$ positive or negative, respectively. With a constant density of states at $\varepsilon=0$, we then have

$$
\overline{G(r=0, \tau)} \sim \rho_{1}(0) / \tau
$$

for large imaginary times. As a result, the uniform superfluid susceptibility, 


$$
\chi \equiv \int d r d \tau \bar{G}(r, \tau),
$$

is actually infinite in the Bose glass phase. This is true despite the fact that average spatial superfluid correlations, $\bar{G}(r, \tau=0)$, decay exponentially. The susceptibility is dominated by rare localized regions which have anomalously low quasiparticle excitation energies. We note in passing that a similar divergent susceptibility occurs also in the Griffiths phase ${ }^{22}$ of the two-dimensional Ising model with bond disorder which is perfectly correlated in one direction, studied by McCoy and $\mathrm{Wu}^{23}$ This model is equivalent to a one-dimensional bond-disordered quantum Ising model in a transverse field at $T=0$. An infinite susceptibility which occurs without long-range order may well exist in Griffiths-like phases of other disordered quantum systems at zero temperature.

By reasoning similar to that for the single-particle density of states, we expect the compressibility $\kappa \equiv \partial \rho / \partial \mu$ to be nonzero throughout the Bose glass phase. This phase therefore differs from the Mott state both by the nonzero compressibility and by an infinite superfluid susceptibility. Nevertheless, both the Mott and Bose glass phases will be insulating, since the spatial superfluid correlations still decay exponentially. For sufficiently large $J$, of course, the system undergoes a transition into the superfluid state, so that the phase diagram consists of three distinct phases.

For the bounded distribution (2.2a) with weak disorder $(\Delta<V / 2)$, there are three possible phase-diagram topologies [Figs. 2(a)-2(c)] consistent with the arguments above; these are distinguished from one another by the occurrence, or lack thereof, of direct Mott insulatorsuperfluid transitions. While it is difficult to infer categorically which of them is correct, one can argue that Fig. 2(c) is extremely unlikely whenever the transitions out of the Mott states are continuous, and that Fig. 2(a) is the most likely. To see the unlikelihood of Fig. 2(c), suppose that the transition out of the Mott insulating state with $\left\langle\hat{\mathbf{N}}_{i}\right\rangle=n$, say, is continuous, i.e., that $\left\langle\hat{\mathbf{N}}_{i}\right\rangle$ changes continuously from the integer $n$ as one moves out of the Mott state by passing through any point on its phase boundary other than the tip. We showed in Sec. II B (the argument remaining valid here) that at any such generic point on the phase boundary there exists a finite energy gap for particle-hole excitations. Hence, the superfluid correlations decay exponentially in the Mott phase, even at the phase boundary. Just slightly outside the phase boundary, one has a small density of extra bosons, $\delta n$, superimposed on the background density state, $n ; \delta n$ is positive (negative) for points in the phase diagram just above (below) the Mott state. In the pure case, these extra particles or holes can move freely through the lattice, thereby producing superfluidity. This is not true in the presence of disorder: Close to the phase boundary, the extra bosons (or holes) are few in number, $\delta n \ll 1$. If, in addition, their typical spacing $(\delta n)^{-1 / d}$ is large compared to the superfluid correlation length of the background state with precisely $n$ bosons per site (i.e., $\delta n=0$ ), it is legitimate to neglect interactions between them mediated by exchange through the background. (One cannot, of course, neglect the direct on-site interaction between the excess bosons.) One may then think of the extra bosons (or holes) as occupying the lowest-lying single quasiparticle (or hole) states of the random effective potential due to the $\delta \mu_{i}$ and the bosons constituting the background Mott state. Since the lowest-lying such states are necessarily localized from the above arguments, the extra particles cannot immediately produce superfluidity. We thus conclude that at any generic point on the phase boundary of the Mott state, the system can only make a continuous transition into an insulating "Bose glass" state rather than a superfluid one, i.e., Fig. 2(c) should not obtain.

The transition from the Mott to Bose glass phase is entirely local-i.e., it is driven by the rare regions of the random potential where a (local) quasiparticle or quasihole gap in vanishing. In this way it is somewhat analogous to the onset of the Griffiths phase in classical random magnets, ${ }^{22}$ in which rare regions are below their effective local transition temperature. However, in contrast to the usual classical case, the properties of the Griffiths phase in the zero-temperature quantum problem are, as we have seen, very different from those of the Mott phase (e.g., the uniform superfluid susceptibility is infinite in the Bose glass phase).

It is more difficult to rule out Fig. 2(b), since at the tip of the Mott phase boundary the gap for producing particle-hole excitations vanishes. It seems possible, therefore, that as one passes out of the Mott state through the tip, the presence of particle-hole excitations allows bosons to hop through the entire system, producing superfluidity immediately, i.e., the phase diagram of Fig. 2(b). This relies implicitly, though, on the (somewhat unlikely) assumption that the initially dilute particle-hole pairs are not themselves localized and hence effectively immobile. One expects rather that the lowest lying particle-hole excitations will be either bound excitons which are localized by the randomness or else appear as unbound (and separate) quasiparticle and quasihole excitations (in equal number), both of which are localized. Therefore, Fig. 2(b) also appears unlikely. At any rate, phase diagram [Fig. 2(b)], if it occurs at all, can only occur for sufficiently weak disorder.

To see this, note that as $\Delta$ approaches $V / 2$ from below, the Mott state shrinks, disappearing when $\Delta=V / 2$. (It is easy to show that, precisely at $\Delta=V / 2$, the compressibility ceases to vanish even in the decoupled limit, $J=0$, where it assumes a positive, constant value for all positive $\mu$ ). At $\Delta=V / 2$, therefore, all trace of the Mott state has vanished, but our earlier arguments demonstrating the insulating nature of the disordered system for all sufficiently small $J$ continue to hold. Thus the phase diagram for $\Delta=V / 2$ is as shown in Fig. 3, viz., Bose glass and superfluid phases occurring for small and large $J / V$, respectively. To be consistent with this limit, the phase diagram for $\Delta$ just slightly less than $V / 2$ must look like Fig. 2(a), rather than Fig. 2(b). If, then, Fig. 2(b) obtains for sufficiently small $\Delta$, there must be a critical value of $\Delta$ above which it reverts to Fig. 2(a). The most likely scenario is that Fig. 2(a) simply holds everywhere.

One more feature of Figs. 2 merits comment, viz., the fact that for densities $\left\langle\hat{\mathbf{N}}_{i}\right\rangle$ which approach 0 (i.e., the 


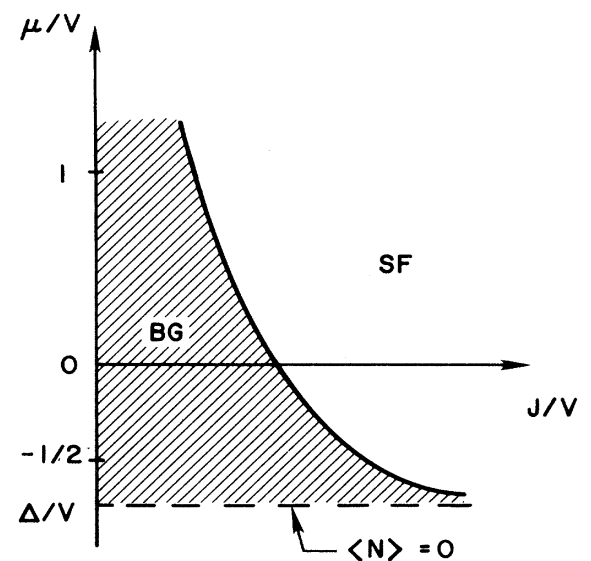

FIG. 3. Zero-temperature phase diagram for the Hamiltonian (2.1) with strong or unbounded disorder. Note that the Mott insulating phase is absent and the superfluid transition is from the Bose glass phase.

negative $\mu$ region of Figs. 2), the Bose glass-superfluid phase boundary moves out to arbitrarily large values of $J$. This is, again, a consequence of the lowest-lying singleparticle levels of a random potential being localized: As $\left\langle\hat{\mathbf{N}}_{i}\right\rangle$ becomes small, only a very few of these low-lying levels are occupied; it takes a large $J$ to assure that at least one of them is extended, i.e., that the system is superfluid.

From the foregoing discussion of the phase diagrams it is clear that for the strongly disordered case $(\Delta>V / 2)$ of distribution (2.2a) the phase diagram is simple: Even in the decoupled limit, $J=0,\left\langle\widehat{\mathbf{N}}_{i}\right\rangle$ varies continuously with $\mu$ for all $\mu$; the average occupation never sticks at integer values. The strong disorder has therefore eliminated the Mott states, leaving only the Bose glass and superfluid phases (Fig. 3). The same qualitative phase diagram is readily seen to describe case $(2.2 b)$ - the Gaussian distribution - as well. This is true even for arbitrarily weak disorder (i.e., small $\widetilde{\Delta}$ ), the unboundedness of the distribution implying the existence, for any $\widetilde{\Delta}$, of sites $i$ with arbitrarily large values of $\left|\delta \mu_{i}\right|$, and hence arbitrarily large numbers of bosons.

The mean-field (infinite-range-hopping) limit of (2.1) remains exactly solvable even in the presence of disorder. The solution, summarized in Appendix A for weak disorder of the form (2.2a) proceeds very much as in the pure case, and results in a similar phase diagram, Fig. 4. It consists of an infinite set of Mott insulating states (corresponding to different integer occupations of the sites), and the superfluid state. As in the pure case, the Mott states are characterized by a gap for particle-hole excitations and zero compressibility. The only real effect of the disorder is to introduce, on the $\mu$ axis, finite gaps between adjacent Mott insulating states. When $\mu$ lies in one of these gaps, any nonzero $J$ produces superfluidity. All other qualitative features (e.g., the fact that for any integer $n$ the $\left\langle\hat{\mathbf{N}}_{i}\right\rangle=n$ line in the superfluid phase joins the Mott insulating state with $\hat{\mathbf{N}}_{i}=n$ at the tip of the lobe) are qualitatively as in the pure system.

The absence of the Bose glass state in the infinite-range

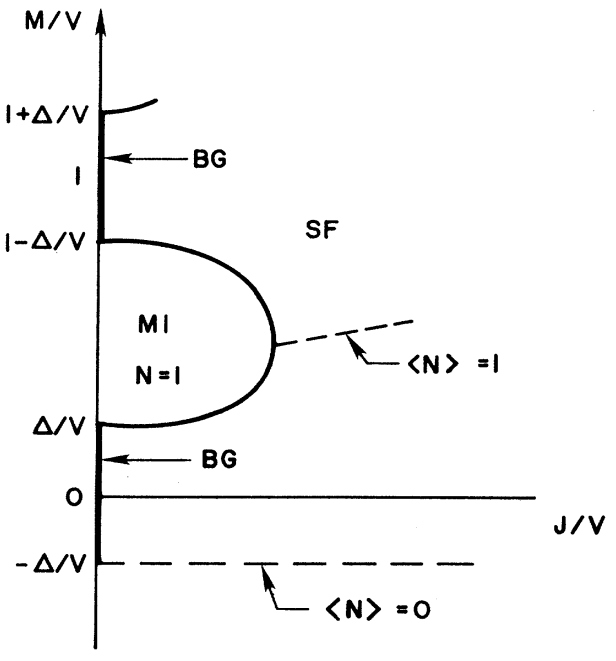

FIG. 4. Portion of phase diagram at zero temperature for weak bounded disorder, obtained from a mean-field treatment of (2.1), exact in the infinite-range hopping limit. In this mean field limit the localized Bose glass phase is unstable to superfluidity for arbitrarily weak hopping $J$.

hopping limit, which was noted previously by $\mathrm{MHL},{ }^{4}$ is readily understood physically: Choose a point in Fig. 4 on the $\mu$ axis between the Mott insulating states with $n$ and $n+1$ particles per site, and consider (as we just did for near-neighbor hopping), the prospect of moving a boson from its position at $J=0$ by turning on a small positive $J$. For simplicity we consider here only the case $n=0$, so that all sites have either one or no bosons (at $J=0$ ), although the argument is easily generalized to arbitrary $n$. Then the easiest bosons to move are obviously the ones with the highest on-site energies, i.e., on the sites with the smallest $\delta \mu_{i}$ 's. The distribution of $\delta \mu_{i}$ 's is continuous, so that there are unoccupied sites arbitrarily close in on-site energy to (and arbitrarily far in space from), the most energetic occupied ones. For any $J \neq 0$, the infinite-ranged hopping thus allows the system to gain kinetic energy with zero cost in (on-site) potential energy by moving particles between these virtually degenerate sites. The bosons free to move in this way are therefore delocalized and hence, since $T=0$, superfluid. Thus the physics of infinite-range hopping in the presence of disorder differs significantly from that of the short-range problem, as pointed out by MHL. ${ }^{4}$ The mean-field limit is therefore a misleading guide to the true phase diagram. It is worth noting that the solvable, infinite-range hopping model corresponding either to distribution (2.2a) with $\Delta>V / 2$, or to the unbounded distribution $(2.2 b)$, is superfluid at zero temperature for all positive values of $J$, again reflecting the absence of the Bose glass phase in the infinite-range limit.

\section{ONSET OF SUPERFLUIDITY: SCALING THEORY}

\section{A. General considerations}

In Sec. II we established the (probable) zerotemperature phase diagrams for a model of lattice bosons 
(2.1), both in the absence and presence of disorder, Figs. $1,2(a)$, and 3 , respectively. In this and the remaining sections, we focus primarily on the behavior of the system in the vicinity of the zero-temperature onset transition to superfluidity. Specifically, in this section we describe a simple scaling theory for the various possible superfluid transitions between the insulating and superfluid phases. Implicit in this development is the assumption that these transitions are in fact continuous, a contention supported by renormalization group results presented in Sec. IV.

Inspection of the phase diagram, depicted in Fig. 1 for the pure case, and Fig. 2(a) or 3 for the disordered case, suggests that there are likely to be three distinct phase transitions to superfluidity: As discussed in Sec. II, in the pure case, superfluid onset from the Mott insulator occurs either as the density moves away from a commensurate value (the pure "generic" transition), or as the hopping strength is increased at fixed commensurate density (the pure fixed-density transition). This latter transition occurs only when the parameters are tuned to sweep through the special multicritical points at the tips of the Mott lobes in Fig. 1. In the disordered case, as argued in Sec. II, we expect that a transition to superfluidity is only possible from the Bose glass phase. This suggests a unique type of superfluid onset transition in the presence of disorder.

To discuss these transitions, it is convenient to introduce a parameter, $\delta$, which is analogous to the reduced temperature $T-T_{c}$ for finite-temperature transitions and thus measures the distance from the transition (which occurs at $\delta=0$ ). For the pure generic (Mott to superfluid) transition and the Bose glass to superfluid transition one can take

$$
\delta \sim \mu-\mu_{c},
$$

with $\mu_{c}$ the chemical potential on the phase boundary. For the special, fixed density, Mott-to-superfluid transition, one must take $\delta \sim J-J_{c}$, since the path of constant (integer) density in the superfluid phase near the tip of the Mott lobe is parallel to the $J$ axis in Fig. 1. One can, however, approach the multicritical point at the tip along a path tangent to the phase boundary, in which case $\delta \sim \mu-\mu_{c}$ remains the appropriate quantity.

The central assumption of our scaling theory is that near the transition there is a single important characteristic length, denoted by $\xi$, which diverges as $\delta^{-v}$ at criticality (this defines $v$ ). At (classical) finite-temperature transitions, static critical phenomena can be discussed with no regard to the system's dynamics, so that $\xi$ is the only important scale. In contrast, the fluctuations at the zero-temperature transitions studied here are purely quantum mechanical, so that static and dynamic quantities are inextricably linked. This necessitates incorporating a characteristic frequency $\Omega$ (and energy $\hbar \Omega$ ) into the scaling description, where $\Omega^{-1}$ is the characteristic time. Generally, one expects $\Omega$ to vanish algebraically at the transition,

$$
\Omega \sim \xi^{-z} \sim \delta^{z v},
$$

which defines the dynamical exponent, ${ }^{24} z$.
The need to incorporate a (diverging) characteristic time into the scaling theory becomes evident when the partition function, $Z=\operatorname{Tr} e^{-\beta \hat{\mathrm{H}}}$, associated with the Hamiltonian $\widehat{\mathbf{H}}$ in (2.1), is expressed as a coherent-state path integral, ${ }^{25}$

$$
\begin{aligned}
& Z=\int \prod_{i} D \Phi_{i}(\tau) D \Phi_{i}^{*}(\tau) e^{-S / \hbar} \\
& S=\int_{0}^{\beta \hbar} d \tau \sum_{i} \Phi_{i}^{*} \hbar \partial_{\tau} \Phi_{i}+\int_{0}^{\beta \hbar} d \tau \hat{\mathbf{H}}\left(\Phi^{*}, \Phi\right) .
\end{aligned}
$$

Here the boson operators in (2.1) have been replaced by $c$-number fields $\Phi_{i}(\tau)$, which depend on both space and imaginary time $\tau$, with periodic boundary conditions:

$$
\Phi_{i}(\tau=0)=\Phi_{i}(\tau=\beta \hbar) .
$$

Since the temporal integration (3.4) runs from 0 to $\beta \hbar$, at $T=0$ the action can be viewed as an effective classical Hamiltonian in $(d+1)$ space-time dimensions. [It should be noted, however, that due to the first term in (3.4) the action will, in general, be complex and anisotropic in the extra dimension.] The (diverging) time scale $\Omega^{-1}$ can then be thought of as a "correlation length" in the $(d+1)$ st dimension, whereas the $T=0$ energy density,

$$
f(\mu, J) \equiv-\lim _{\beta \rightarrow \infty} \lim _{N \rightarrow \infty} \frac{1}{\beta N} \ln Z,
$$

is equivalent to the $(d+1)$-dimensional classical free energy density.

With this analogy to a $(d+1)$-dimensional classical theory, a $T=0$ scaling theory of superfluid onset can readily be formulated. As usual, if hyperscaling obtains the singular part of the energy density, $f_{s}$, satisfies a homogeneity condition near the transition, ${ }^{26}$

$$
f_{s}(\delta) \sim b^{-(d+z)} f_{s}\left(b^{1 / v} \delta\right) \sim \delta^{v(d+z)},
$$

for arbitrary length rescaling parameter $b$. The second equality above follows from the standard choice $b=\delta^{-v}$. Moreover, one expects correlation functions near the transition to exhibit scaling forms. Specifically, consider the order-parameter susceptibility defined as

$\bar{\chi}(r, \tau) \equiv \overline{\left\langle T_{\tau} \Phi_{r}(\tau) \Phi_{0}^{+}(0)\right\rangle}-\overline{\left\langle\Phi_{r}(\tau)\right\rangle\left\langle\Phi_{0}^{+}(0)\right\rangle}$,

where the angular brackets refer to a ground-state expectation value and the overbar denotes an ensemble average over realizations of disorder. In the vicinity of the transition one expects the long-distance and long-time behavior of $\bar{\chi}(r, \tau)$ to be scaled by the correlation length $\xi$ and $\xi^{z}$, respectively, at least up to a background power law,

$$
\bar{\chi}(r, \tau) \sim r^{-(d+z-2+\eta)} g\left(r / \xi, \tau / \xi^{z}\right) .
$$

As usual, the power law at criticality $(\xi=\infty)$ defines the exponent $^{27} \eta$.

For the following discussion it is convenient to consider the effect of adding to the action $S$ in (3.4) a fictitious (fieldlike) term which is linear in the order parameter $\Phi$ :

$$
S \rightarrow S-\int d \tau \sum_{i}\left[h \Phi_{i}^{*}(\tau)+\text { c.c. }\right] .
$$

Since superfluidity is associated with a symmetry break- 
ing, $M=\langle\hat{\boldsymbol{\Phi}}\rangle$, this field term, which explicitly breaks the symmetry, will destroy the transition (i.e., it must be relevant). One thus expects the energy density to scale as $^{26}$

$$
f_{s}(\delta, h) \sim \delta^{v(d+z)} Y\left(h / \delta^{\phi_{h}}\right),
$$

with an appropriate scaling function $Y$ and a positive exponent $\phi_{h}$. This form, when used in conjunction with (3.8), and the fact that

$$
\bar{\chi}(k=0, \omega=0) \sim \partial^{2} f / \partial h^{2},
$$

enables one to generalize the usual hyperscaling relation between $\beta\left(M \sim \delta^{\beta}\right)$ and $\eta$ :

$$
2 \beta=v(d+z-2+\eta) .
$$

Note that, by using (3.12) and Ref. 27, Eq. (3.11) can be rewritten as $\alpha+2 \beta+\gamma=2$. [We remark in passing that since the special Mott-to-superfluid transition at fixed density is a multicritical point (see Fig. 1), one expects that, in addition to $\delta$ and $h$, there is another relevant field at the transition. This is indeed the case; in Sec. IV A this extra relevant field is identified, and the associated crossover exponent computed.]

Scaling forms for some physical observables follow directly from the energy (3.6) or (3.10). For example, from (3.4) it is apparent that the compressibility, $\kappa \equiv \partial \rho / \partial \mu$ is given by $\kappa=-\partial^{2} f / \partial \mu^{2}$. Thus, provided $\delta \sim \mu-\mu_{c}$, as in (3.1), the singular part of the compressibility, $\kappa_{s}$, follows from (3.6) by differentiation, scaling near the transition as

$$
\kappa_{s} \sim \delta^{v(d+z)-2} \equiv \delta^{-\alpha} .
$$

This generalizes the usual hyperscaling expression $\alpha=2-d v$. Here the compressibility is the $T=0$ analog of the specific heat. It should be emphasized that the scaling form (3.12) does not apply at the special (multicritical) fixed-density Mott-to-superfluid transition, for which, as noted after (3.1), one must take $\delta \sim J-J_{c}$; differentiation with respect to $\delta$ thus becomes inequivalent to differentiation with respect to chemical potential.

\section{B. Generalized Josephson relations: superfluid density and compressibility}

For boson systems where the order parameter itself is difficult to observe directly, an important, experimentally observable, physical quantity is the superfluid density, $\rho_{s}$. Since $\rho_{s}$ is a measure of the spatial stiffness of the phase, $\phi$, of the (complex) order parameter and not the order parameter $\langle\Phi\rangle$ itself, its scaling behavior does not follow immediately from (3.6) to (3.8) and thus requires separate consideration. In this subsection we derive hyperscaling relations for the singular behavior of both the superfluid density and the total compressibility.

Strictly speaking, the superfluid density is defined via the change in energy (or free energy at $T \neq 0$ ) of a system under a change in boundary condition which imposes a twist (by say $\pi$ ) of the phase of the order parameter across the system. ${ }^{11}$ More loosely, $\rho_{s}$ is proportional to the coefficient of $(\nabla \phi)^{2}$ in a long-wavelength effective action (or Hamiltonian) which describes (only) the slow variations in the phase of the order parameter, in the superfluid state. Since at $T=0$ the order parameter depends on both space and (imaginary) time in (3.4), one expects this effective Hamiltonian to depend on $\phi(\tau) \equiv \partial \phi / \partial \tau$ as well. Indeed, the long-wavelength, lowenergy effective action describing the superfluid phase can be expressed quite generally as an expansion in powers of $\nabla \phi$ and $\phi$, namely, ${ }^{28}$

$$
\begin{aligned}
& S_{\mathrm{eff}}=\frac{1}{2} \int d^{d} x \int_{0}^{\beta \hbar} d \tau[ 2 \rho(i \hbar \dot{\phi})+\kappa(\hbar \dot{\phi})^{2} \\
&\left.+\left(\hbar^{2} \rho_{s} / m\right)(\nabla \phi)^{2}\right],
\end{aligned}
$$

where $\rho$ is the average density. Terms involving higherorder derivatives $(\nabla \phi)^{3}$ and $\phi^{3}$, have been ignored. Notice that the (boundary) term linear in $\phi$ has been chosen complex (see the following). The quadratic terms describe second sound (or third or fourth sound for thin films or porous media, respectively) in the superfluid with velocity $C_{2}$ satisfying

$$
C_{2}^{2}=\rho_{s} / m \kappa,
$$

with $m$ the real physical mass of the bosons.

We now argue that the (constant) coefficients $\rho$ and $\kappa$ in (3.13) are in fact the full exact density and compressibility, respectively. This follows most easily from the invariance of the action (3.4) under the transformation consisting of a shift in the chemical potential and a corresponding (spatially-uniform) change of phase of the order parameter $\Phi$ :

$$
\begin{aligned}
& \mu \rightarrow \mu^{\prime}=\mu+i \delta \mu, \\
& \Phi(x, \tau) \rightarrow \Phi^{\prime}(x, \tau)=\Phi(x, \tau) \exp (i \delta \mu \tau / \hbar) .
\end{aligned}
$$

[Note that this invariance is equivalent, when expressed in terms of the real time variable $t \equiv i \tau$, to the familiar Josephson relation, $\delta \mu=\hbar \partial \phi(t) / \partial t$, between the time derivative of the order-parameter phase, $\phi(t)$, and changes in the chemical potential.] Now imagine imposing boundary conditions at $\tau=0$ and $\tau=\beta \hbar$ to enforce a total twist of $\Delta \phi$, say, in the phase $\phi(\tau)$ over the range $0 \leq \tau \leq \beta \hbar$. Equations (3.15) imply that the resulting problem can be transformed into one wherein there is no twist of $\phi(\tau)$, but where the chemical potential $\mu$ has been shifted by $\mu \rightarrow \mu^{\prime}=\mu-i \Delta \phi / \beta$. The change, $\delta f$, in energy density resulting from the twist can thus be computed straightforwardly as a Taylor series in $\Delta \phi$ :

$$
\begin{aligned}
\delta f= & (\partial f / \partial \mu)(-i \Delta \phi / \beta) \\
& +\frac{1}{2}\left(\partial^{2} f / \partial \mu^{2}\right)(-i \Delta \phi / \beta)^{2}+\cdots .
\end{aligned}
$$

Since the density, $\rho$, and compressibility, $\kappa$, are defined as $-\partial f / \partial \mu$ and $-\partial^{2} f / \partial \mu^{2}$, respectively, and since imposition of the twist $\Delta \phi$ induces a time derivative, $\dot{\phi}$, of $\phi$, with average value $\dot{\phi}=\Delta \phi / \beta \hbar,(3.16)$ can be rewritten in precisely the form (3.13); this demonstrates that $\rho$ and $\kappa$ are indeed the correct coefficients in (3.13), and, moreover, that the linear term in $\dot{\phi}$ is imaginary. 
The effective action (3.13) can now be employed to obtain scaling forms for both the superfluid density and total compressibility. The most straightforward approach employs a finite-size scaling form for the energy density,

$$
f_{s}(\delta, L, \beta) \sim \delta^{v(d+z)} Y\left(\xi / L, \xi^{z} / \beta\right),
$$

for a system of spatial size $L^{d}$ and temporal extent $\beta$. Again, imagine imposing through boundary conditions at $\tau=0$ and $\beta \hbar$ a total twist, $\Delta \phi$, in the order parameter phase. It is apparent from (3.13) that the excess energy density needed to enforce the twist $\Delta \phi=\pi$, relative to $\Delta \phi=0$, varies as $\delta f \sim \kappa / \beta^{2}$ for $L, \beta \rightarrow \infty$. Alternatively, one has from (3.17),

$$
\Delta f \sim \delta^{(d+z) v} \widetilde{Y}\left(\xi / L, \xi^{z} / \beta\right),
$$

where $\widetilde{Y}$ is the difference between finite-size scaling functions at $\Delta \phi=\pi$ and 0 (i.e., $\widetilde{Y}=Y_{\pi}-Y_{0}$ ). ${ }^{29}$ In order that these two expressions for $\delta f$ be equal, $\widetilde{Y}$ must behave like $\left(\xi^{z} / \beta\right)^{2}$ as $L$ and $\beta \rightarrow \infty$, where upon $\kappa \sim \xi^{2 z} \delta^{(d+z) v}$. An analogous argument, imposing a twist in space instead of time, yields a scaling form for the superfluid density $\rho_{s} \sim \xi^{2} \delta^{(d+z) v}$, a result obtained recently by MHL. ${ }^{4} \mathrm{Fi}-$ nally, the fact that $\xi \sim \delta^{-v}$ near the transition enables one to rewrite these scaling equations as

$$
\begin{aligned}
& \rho_{s} \sim \delta^{\zeta}, \text { with } \zeta=v(d+z-2), \\
& \kappa \sim \delta^{v(d-z)} .
\end{aligned}
$$

These generalized Josephson relations are the central results of this section.

It is instructive to rederive the hyperscaling relations more formally by employing the scaling form for the order-parameter susceptibility $\bar{\chi}$ (3.8), in conjunction with the effective action (3.13). Specifically, in the superfluid phase one may evaluate $\bar{\chi}(r, \tau)$ for $r \gg \xi$ and $\tau \gg \xi^{z}$ by replacing $\Phi(r, \tau)$ in (3.7) with $\Phi_{0} \exp [i \phi(r, \tau)]$ (i.e., ignoring amplitude fluctuations) and evaluating the expectation values using the action (3.13). This yields

$$
\bar{\chi}(r, \tau) \simeq M^{2}\left\{\exp \left[\langle\phi(r, \tau) \phi(0,0)\rangle_{\mathrm{eff}}\right]-1\right\},
$$

where \langle\rangle$_{\text {eff }}$ denotes an average with respect to (3.13) and $M \equiv\langle\Phi\rangle$. For $d \geq 1$ the argument of the exponential in (3.19) decays to zero for $r, \tau \rightarrow \infty$. Thus one obtains

$$
\begin{aligned}
\bar{\chi}(k, \omega) & \simeq M^{2}\left\langle|\phi(k, \omega)|^{2}\right\rangle_{\mathrm{eff}} \\
& =M^{2}\left[\hbar \kappa \omega^{2}+\left(\hbar \rho_{s} / m\right) k^{2}\right]^{-1} ; \quad k \xi \ll 1, \quad \omega \xi^{2} \ll 1 .
\end{aligned}
$$

Alternatively, a scaling form for $\bar{\chi}(k, \omega)$ can be obtained directly by Fourier transforming (3.8), namely,

$$
\bar{\chi}(k, \omega) \sim \xi^{2-\eta} \widetilde{g}\left(k \xi, \omega \xi^{z}\right),
$$

with an appropriate scaling function $\widetilde{g}$. Then, requiring that (3.20) and (3.21) give the same $\omega$ dependence for $\omega \xi^{z}<<1$ and $k=0$, implies that $\widetilde{g}(0, y) \sim 1 / y^{2}$ for $y \rightarrow 0$. Moreover, equating coefficients of the $1 / \omega^{2}$ terms gives

$$
\kappa \sim M^{2} \xi^{2 z+\eta-2} \sim \delta^{2 \beta+v(2-\eta-2 z)} .
$$

Finally, upon using the hyperscaling expression (3.11) for $\beta$, one arrives at the compressibility hyperscaling expression (3.18b). The superfluid density scaling relation (3.18a) is obtained similarly, through the equality of the two expressions for $\bar{\chi}(k, \omega)$ at $\omega=0$ and $k \xi \ll 1$.

\section{Critical exponents}

In the remainder of this section we demonstrate that nontrivial information concerning the exponents $v$ and $z$ can be extracted by comparison of the scaling relations for the singular part of the compressibility, $\kappa_{s}$ (3.12), and the total compressibility, $\kappa$ (3.18). Moreover, consideration of the single-particle density of states near and at criticality indicates a probable (upper) bound on $\eta$ for the Bose glass to superfluid transition. These results are confirmed and extended by the renormalization-group (RG) calculations of the following section.

\section{Pure system}

Since the hyperscaling equation for $\kappa_{s}$ in (3.12) does not hold for the special fixed-density Mott to superfluid transition, we exclude this case from the present discussion and focus on the generic Mott to superfluid transition and, in the disordered case, on the Bose glass to superfluid transition.

Consider first, then, the generic transition between the Mott insulator and superfluid in the pure case. Since the Mott phase is incompressible $(\kappa=0)$, the total compressibility cannot have an analytic piece at the transition. Thus the exponent for the singular part of the compressibility on the superfluid side, (3.12), can be equated with that for the total compressibility, (3.18b), giving

$$
z v=1 \text {. }
$$

This exponent equality should hold for all dimensions $d$ such that the hyperscaling relations for $\kappa$ and $\kappa_{s}$ are not violated, i.e., for $d$ less than the upper critical dimension, $d_{c}$. By analogy with the superfluid onset transition at $T \neq 0$, one expects that even at $T=0, \zeta$ and $v$ are fixed at their mean field values of 1 and $\frac{1}{2}$, respectively, for all $d>d_{c}$. Using (3.22) and the hyperscaling for $\rho_{s}(3.18 \mathrm{a})$, one thereby deduces that

$$
d_{c}=2
$$

for the generic Mott insulator to superfluid transition. This result can be verified by direct $R G$ calculation (Sec. IV A), which also demonstrates that although the hyperscaling expressions (3.18) for $\kappa$ and $\rho_{s}$ are violated for $d>2$, the exponent equality (3.22) continues to hold in arbitrary dimension.

Note that one can approach the multicritical point at the tip of any Mott lobe in Fig. 1 along a path of constant $J$. On such a path, which is tangent to the phase boundary at the multicritical point, $\delta \sim \mu-\mu_{c}$, so that (3.12) continues to hold. (Recall that (3.12) does not apply for the path of fixed integer density.) Let us assume that $\kappa$ approaches zero in a singular fashion as $\delta \rightarrow 0$ along this path (the other alternative, given that $\kappa$ is positive in the superfluid phase, being $\kappa \sim \delta^{2}$ as $\delta \rightarrow 0$ ). As in the generic case, one can then equate (3.12) and (3.18b), thereby ob- 
taining $\widetilde{v} \widetilde{z}=1$, i.e., Eq. (3.22). Here we use a tilde to denote exponents along this special path. Again, this relation should hold for all $d$ less than the upper critical dimension, $d_{c}$, for the multicritical point. Noting (see Sec. IV A) that the mean-field values of $\zeta$ and $v$ for the constant- $J$ approach to the multicritical point are 2 and 1 , respectively, and combining these results with $(3.18 \mathrm{a})$ and (3.22) one deduces $d_{c}=3$ for the multicritical point. Confirmation of this result, together with a demonstration that (3.22) holds on the constant- $J$ path for all $d$, is given in Sec. IV A; our assumption that $\kappa$ vanishes in a singular manner along this path is therefore justified. It is worth emphasizing that (3.12), and hence (3.22), are only valid along asymptotically tangent paths (e.g., not along the fixed-density trajectory) to the multicritical point.

\section{Bose glass-superfuid transition}

Consider next the Bose glass to superfluid transition in the disordered case. Since the total compressibility $\kappa$ is finite and nonzero in both the insulating Bose glass and the superfluid phases (cf. Sec. II), it seems plausible that $\kappa$ is also finite (and nonzero) right at the transition. It seems, in particular, rather unreasonable that $\kappa$ should vanish at the transition and so open up a pseudo gap, given that it is positive in the two states. The finiteness and nonvanishing of $\kappa$ at criticality would necessarily imply, ${ }^{30}$ from $(3.18 \mathrm{~b})$, that $z=d$. We argue below that this is indeed the case.

Suppose for the moment that $z>d$ so that $\kappa$ diverges at the transition. Then, as in the pure case, one can equate the exponents of $\kappa$ and $\kappa_{s}$, giving $z v=1$, which (when combined with $z>d$ ) implies the inequality $v<1 / d$. However, a theorem proved recently by Chayes et al., ${ }^{12,31}$ states that

$$
v \geq 2 / d
$$

for all $d$ for any transition which can be controlled by varying the strength of (spatially uncorrelated) disorder. Since the Bose glass to superfluid transition falls into this class, one arrives at a contradiction. Hence the initial assumption, $z>d$, must be false. Thus, provided the hyperscaling expressions for $\kappa$ and $\kappa_{s}$ are valid, the total compressibility cannot diverge at the transition.

Similar reasoning under the assumption of vanishing compressibility $(z<d)$, implies that $v=1 / z \geq 2 / d$, or $z \leq d / 2$. However, the physical requirement that the second sound speed, $C_{2}$, in (3.14), must not diverge at $\delta=0$ implies $z \geq 1$ for all $d$, since [from (3.18)] $C_{2} \sim \delta^{v(z-1)}$. Thus, at least for $d<2$, the only remaining possibility is

$$
z=d \text {, }
$$

implying that the total compressibility is finite at the transition. In this case $\kappa_{s}$ represents only a singular correction to the total compressibility (with exponent $\geq 2$ ) so that (3.22) need not be satisfied. It will be shown in Sec. IV C that, in the one-dimensional case, $z=d=1$ and the compressibility is finite at the transition, consistent with (3.25). As mentioned above, on physical grounds we expect that $\kappa$ will not vanish at criticality (even for $d \geq 2$ ) implying the equality $z=d$ in arbitrary dimension $d$.

One might worry, however, that above some upper critical dimension, $d_{c}$, hyperscaling would be violated and the equality (3.25) break down. Typically, for $d>d_{c}$ hyperscaling breaks down due to the presence of a dangerous irrelevant operator associated with a stable Gaussian fixed point (with associated mean-field exponents $v=\frac{1}{2}$ and $\xi=1$ ). Were this to happen for the Bose glass to superfluid transition, one could deduce $d_{c}$ by combining the equality $z=d$ (for $d \leq d_{c}$ ) with the requirement that $\zeta=1$. This gives $d_{c}=2$, as in the pure case (3.25). However, in the presence of disorder, $v=\frac{1}{2}$ in $d=2$ is forbidden by the general theorem (3.24); the possibility of a conventional onset of mean-field theory, in which the critical exponents smoothly approach their mean-field values as some upper critical dimension is approached from below, is therefore eliminated. In light of this, the simplest conclusion is that for the Bose glass to superfluid transition the compressibility is finite, and the equality $z=d$ holds for all $d \geq 1$. An alternative possibility, that for weak disorder the exponents jump discontinuously to their mean-field values at $d_{c}=4$, is outlined in Sec. IV C and discussed in detail by Weichman and $\mathrm{Kim}$ in Ref. 8. This scenario is consistent with the inequality (3.24) which, since $v=\frac{1}{2}$ in mean-field theory, requires $d_{c} \geq 4$.

Having obtained nontrivial information concerning the exponents $v$ and $z$ for the Bose glass to superfluid transition, we now focus on the remaining independent exponent $\eta$, and derive an upper bound. To this end, consider the single-particle density of states, $\rho_{1}(\varepsilon)$, defined in (2.6), and related by Laplace transform to the imaginary time Green's function $\bar{G}(r=0, \tau)$ in (2.7). The behavior of $\rho_{1}(\varepsilon)$ near the transition can be obtained from the scaling of $\bar{G}(r=0, \tau)$ deduced from (3.8):

$$
\bar{G}(r=0, \tau) \sim \tau^{-(d+z-2+\eta) / z} \widetilde{g}\left(\tau / \xi^{z}\right)
$$

in the Bose glass phase, and $\bar{G}(r=0, \tau \rightarrow \infty)=M^{2}$ in the superfluid phase. In (3.26) $\widetilde{g}(x \rightarrow 0)$ tends to a nonzero constant.

As emphasized in Sec. II, $\rho_{1}(\varepsilon)$ is expected to approach a nonzero constant as $\varepsilon \rightarrow 0$, throughout the entire Bose glass phase, so that $\bar{G}(r=0, \tau) \sim 1 / \tau$ for $\tau \rightarrow \infty$. This determines the large $x$ behavior of the scaling function $\widetilde{g}(x)$ and implies, upon using (2.8), that in the Bose glass phase $\rho_{1}(\varepsilon=0) \sim \xi^{2-d-\eta}$ near criticality. Right at the transition, on the other hand, we have

$$
\rho_{1}(\varepsilon) \sim \varepsilon^{(d-2+\eta) / z} .
$$

In the superfluid phase, since $\bar{G}(r=0, \tau \rightarrow \infty)=M^{2}$, the single-particle density of states has a $\delta$-function contribution at $\varepsilon=0: \rho_{1}(\varepsilon) \sim M^{2} \delta(\varepsilon)$. This is due to enhanced absorption (and emission) from the condensate.

On physical grounds we expect that as the transition is approached from the Bose glass phase, $\rho_{1}(\varepsilon=0)$ will grow due to precursor superfluid fluctuations, and diverge at criticality (to match onto $\rho_{1}(\varepsilon) \sim \delta(\varepsilon)$ in the superfluid phase). This implies that $\eta$ must satisfy the bound 


$$
\eta \leq 2-d \text {. }
$$

A trivial lower bound can also be obtained by the requirement that the exponent $d+z-2+\eta$ in (3.26) be positive. With $z=d$ this implies $\eta>2-2 d$.

Although the argument used to obtain (3.27) seems entirely plausible, we cannot completely rule out the possibility that $\rho_{1}(\varepsilon=0)$ is discontinuous at criticality. It will be shown in Sec. IV, however, that in the onedimensional case $\eta=\frac{2}{3}$, satisfying the bound (3.27), and implying that $\rho_{1}(\varepsilon=0)$ diverges smoothly from the insulating side.

Before closing this section, it is instructive to compare our conclusions for the Bose glass to superfluid transition with corresponding results for the $(T=0)$ metal-insulator transition in disordered Fermi systems. ${ }^{1}$ Both with and without (short-range) electron-electron interactions, the total compressibility is believed to be finite at the metalinsulator transition. ${ }^{1}$ This similarity between the Bose and Fermi transitions is perhaps hardly surprising: indeed, provided hyperscaling holds at the metalinsulator transition, the argument presented after (3.24) to rule out a divergent compressibility in the Bose case, applies directly to the Fermi case as well, at least when interactions are present. In the noninteracting fermion case (Anderson localization) the dynamic exponent $z$ is equal to $d$, precisely as we deduced for the Bose case, and the transition has a well-developed field theoretic description near $d=2$. Yet, as in the Bose case, there is little evidence for mean-fieldlike behavior in high dimensions. Indeed, it seems likely that no upper critical dimension exists (i.e., $d_{c}=\infty$ ). In addition, for Anderson localization the exponent $\eta=2-d$ due to a constant density of states at the mobility edge. ${ }^{1}$ This value saturates the upper bound (3.27) for the Bose case, where we expect the density of states to diverge at criticality. The striking similarity between the Bose and Fermi results suggests that the arguments described in this section might lead to insight into the Fermi problem. For both fermions and bosons, though, the long-range Coulomb interaction requires a generalization of the above picture. Preliminary analysis suggests that, for bosons with a $1 / r$ interaction, the dynamic exponent $z$ is equal to one for all $d$. This should be relevant to disordered superconductors undergoing a transition from an insulator to a superconductor.

\section{CRITICAL PHENOMENA AT THE ONSET OF SUPERFLUIDITY}

This section is devoted to a more quantitative study of the critical phenomena associated with the zerotemperature superfluid onset. The renormalization-group calculations described here confirm and supplement the scaling theory of Sec. III. In Sec. IV A we consider the pure case, deriving the field theory which describes both the generic Mott insulator to superfluid transition and the special multicritical point (Fig. 1), and so identifying the associated universality classes. Section IV B is devoted to a discussion of the one-dimensional problem, both with and without disorder. In particular, the Bose glass to superfluid transition, studied recently by Giarmarchi and Schulz, is reviewed in light of the general scaling theory of Sec. III. Finally, in Sec. IV C we describe the difficulties one encounters when trying to analyze the critical properties at the onset of superfluidity in high dimensions, in the presence of disorder. A brief critique is given of a recent attempt by $\mathrm{MHL}^{4}$ along these lines.

\section{A. Lattice bosons with no disorder}

To study the critical phenomena associated with the transitions in Fig. 1, it is useful to generate, from the original Hamiltonian (2.1), a field theoretic representation of the partition function. A convenient procedure is detailed in Appendix A: Expressing the partition function, $Z=\operatorname{Tr} \exp (-\beta \hat{\mathbf{H}})$, in the interaction representation, one can decouple the off-site hopping term $\hat{\mathbf{H}}_{1}$ by means of a Hubbard-Stratanovich transformation, ${ }^{9,10}$ introducing a complex $c$-number field $\psi_{i}(\tau)$. In this way the partition function is expressed as

$$
Z=Z_{0} \int \prod_{i} D \psi_{i}(\tau) D \psi_{i}^{*}(\tau) \exp [-S(\psi)]
$$

with an effective action,

$$
S(\psi)=\sum_{i, j} \int d \tau\left(J^{-1}\right)_{i j} \psi_{i}^{*}(\tau) \psi_{j}(\tau)-\sum_{i} \ln \left\langle T_{\tau} \exp \left[\int d \tau \psi_{i}(\tau) \hat{\boldsymbol{\Phi}}_{i}^{+}(\tau)+\text { H.c. }\right]\right\rangle_{0},
$$

where $\left(J^{-1}\right)_{i j}$ is the inverse of the matrix of hopping coefficients. In (4.2) the average is taken in the ensemble corresponding to $\hat{\mathbf{H}}_{0}$. Since $\hat{\mathbf{H}}_{0}$ does not couple field operators $\hat{\boldsymbol{\Phi}}_{i}$ at different sites, $i$, this average can be easily evaluated as a cumulant expansion in powers of $\psi_{i}$. Since for small $\left\langle\hat{\boldsymbol{\Phi}}_{i}\right\rangle,\left\langle\psi_{i}\right\rangle$ is linearly related to $\left\langle\hat{\boldsymbol{\Phi}}_{i}\right\rangle$, the field $\psi_{i}$ serves as an order parameter for superfluidity.

To extract the critical behavior one must retain both the spatial and temporal dependence of the auxiliary field $\psi_{i}(\tau)$ in (4.2). To this end, it is sufficient to perform a spatial gradient expansion in the first term, and in the second term a temporal gradient expansion, as well as the cumulant expansion in powers of $\psi$. Retaining only the most relevant terms, and replacing the spatial lattice by a continuum with a high momentum cutoff, one finds

$$
S=\frac{1}{2} \int_{k, \omega}\left(k^{2}+\omega^{2}+r\right)|\psi(k, \omega)|^{2}+g \int_{k, \omega} i \omega|\psi(k, \omega)|^{2}+u \int_{x, \tau}|\psi|^{4} .
$$

Here $x$ and $\tau$ have been rescaled to fix the coefficients of the $k^{2} \psi^{2}$ and $\omega^{2} \psi^{2}$ at $\frac{1}{2}$. In the pure case considered in this subsection the parameters $r, g$, and $u$ are constants, independent of $x$.
Note that the action (4.3) is real except for the $g i \omega \psi^{2}$ operator. If $\psi$ is viewed as a two-vector, $\psi=\left(\psi_{1}, \psi_{2}\right)$, this term takes the form of a cross product: $g\left(\psi_{1} \partial_{1} \psi_{2}-\psi_{2} \partial_{\tau} \psi_{1}\right)$. The other terms shown can be writ- 
ten as dot products. The coupling constant $g$, when expressed in terms of the parameters in the original model (2.1), takes the form

$$
g=g(\mu, J)=\partial r(\mu, J) / \partial \mu,
$$

with $r(\mu, J)$ given explicitly in (A6). Since in mean-field the phase boundary is given by the condition $r(\mu, J)=0$, $g$ vanishes precisely at the multicritical point. Points on the phase boundary above (below) this point correspond to positive (negative) $g$, reflecting the absence of particlehole symmetry. Presumably this same feature continues to hold when fluctuations are included (see the arguments in II B).

As discussed in Sec. III, one expects the $T=0$ superfluid onset transition to be characterized by both a diverging length scale $\xi \sim \delta^{-v}$ and a diverging time scale $\Omega^{-1} \sim \delta^{-z v}$, where $\delta \equiv r-r_{c}$ measures the distance to the transition and $z$ is the dynamic exponent. Moreover, excitation energies which vanish at criticality should scale with $\hbar \Omega$. Thus, for example, in the insulating regime the Mott gap should vanish as $E_{g} \sim \delta^{z v}$.

\section{Multicritical point}

We first analyze the multicritical point at the tip of each Mott lobe. For $g=0$, (4.3) becomes isotropic in space and time; it is simply the soft-spin representation of the $(d+1)$-dimensional $X Y$ model. Hence the multicritical point $(g=\delta=0)$ is described by the $X Y$ fixed point in $d+1$ dimensions. The lower and upper critical dimensions are therefore $d_{l}=1$ and (as inferred from scaling in the previous section) $d_{c}=3$, respectively. The space-time isotropy implies that $z=1$, so that $E_{g}$ vanishes with the $(d+1)$-dimensional $X Y$ correlation length exponent, $v_{X Y}$. Thus for $d \geq 3$, where $v_{X Y}=\frac{1}{2}$, the phase boundary is parabolic at the multicritical point, as depicted in Fig. 1.

The $X Y$ multicritical point is best understood through the $\mathrm{RG}$ scaling relation for the singular part of the energy density, $f_{s}$, for small $\delta\left(\equiv r-r_{c}\right)$ and $g$, and $d \leq d_{c}$ :

$$
f_{s}(\delta, g) \sim b^{-(d+z)} f_{s}\left(\delta b^{1 / v_{X Y}}, g b^{\lambda_{g}}\right),
$$

or, equivalently,

$$
f_{s} \sim \delta^{(d+z) v_{X Y}} \widetilde{f}_{s}\left(g / \delta^{\phi_{g}}\right) .
$$

Here $z=1, b(>1)$ is the rescaling parameter, and the $\mathrm{RG}$ eigenvalue, $\lambda_{g}$, of the operator $i \omega \psi^{2}$ at the multicritical point, is related via $\phi_{g} \equiv \lambda_{g} v_{X Y}$ to the crossover exponent $\phi_{g}$ which controls the crossover from the multicritical point to the generic Mott-superfluid transition; the scaling function $\widetilde{f}_{S}(x)$ behaves like a constant or like $x^{(d+z) / \lambda_{g}}$ as $x$ approaches 0 or $\infty$ respectively, in order that $f_{s}$ be finite and nonzero in both these limits; for $\delta<0, \widetilde{f}_{s}(x)$ is singular for some $x_{c}$ with a singularity characteristic of the generic Mott-superfluid critical point discussed below. The eigenvalue $\lambda_{g}$ can be computed perturbatively in powers of $\varepsilon=3-d$, directly from the action (4.3); one finds $\lambda_{g}=1+O\left(\varepsilon^{2}\right)$. One can, however, deduce quite generally, even in the presence of disorder, that $\lambda_{g}$ is identically $z(=1$ in the pure system) to all or- ders in $\varepsilon$, by noting that the action (4.3) describes a classical $(d+1)$-dimensional superfluid in an external gauge field $\mathbf{A} \equiv\left(\mathbf{A}_{\perp}, A_{\tau}\right)=(0, i g)$. Then, since ig can be shifted away by a generalization of the Josephson relation (3.15), at the expense of a temporal twist, the second derivative

$$
\partial^{2} f / \partial A_{\tau}^{2} \sim \delta^{v_{x y}\left(d+z-2 \lambda_{g}\right)}
$$

represents a temporal superfluid density, $\rho_{s}^{\tau}$. A finite size scaling argument, identical to that leading to $(3.18 \mathrm{~b})$, yields $\rho_{s}^{\tau} \sim \delta^{v_{x y}(d-z)}$, from which follows $\lambda_{g}=z$. Hence, $\phi=z v_{x y}$ is large even for $d<3$.

Equations (4.4) make clear that the properties of the multicritical point depend on the direction from which it is approached. Since $r$ and $g$ are presumably analytic functions of the underlying microscopic variables $\mu$ and $J, g \sim \delta$ as one approaches the multicritical point along a typical path in the superfluid phase in Fig. 1. Since $v_{X Y}<1$ for all $d+1 \geq 3$, the argument of $\widetilde{f}_{s}$ in $(4.4 \mathrm{~b})$ must therefore vanish as the multicritical point is approached along such paths, for $2 \leq d \leq 3$. But $\widetilde{f}_{s}(x) \rightarrow$ constant as $x \rightarrow 0$, so in this typical approach, $g$ drops out of the scaling relation $(4.4 \mathrm{~b})$, and the multicritical point looks like an ordinary $(d+1)$-dimensional $X Y$ critical point. This is certainly true, for example, for the approach to the multicritical point along the path of fixed integer density, and implies that critical exponents such as $\beta$ and $\eta$ assume $(d+1)$-dimensional $X Y$ values.

In the special case where the path of approach is asymptotically tangent to the phase boundary (i.e., to the $\mu$ axis) of Fig. 1, however, the situation is different. On such paths, which pass through the multicritical point but do not penetrate into the Mott phase, $\delta \sim r-r_{c}$ is always non-negative; presumably, then, $\delta \sim\left(\mu-\mu_{c}\right)^{2}$, while $g$ is simply proportional to $\mu-\mu_{c}$, i.e., $\delta \sim g^{2}$. Since $v_{X Y}>\frac{1}{2}$ for $d<d_{c}=3$, the argument of $f_{s}$ in (4.4b) blows up as $g \rightarrow 0$, whereupon $f_{s} \sim g^{d+z}=g^{d+1}$. Similar arguments allow the computation of the other exponents along this special path, which we denote by a tilde, with the results $\widetilde{\eta}=\eta_{X Y}, \widetilde{v}=\widetilde{z}=1, \xi=d-1$, etc. As usual, these exponents stick at their mean-field values, $\widetilde{z}=1$, $\widetilde{v}=1, \tilde{\eta}=0, \widetilde{\xi}=2, \widetilde{\alpha}=-2$, etc., for $d \geq d_{c}=3$. The mean-field values follow directly from the observation that

$$
\delta=r-r_{c} \sim g^{2} \sim\left(\mu-\mu_{c}\right)^{2}
$$

on the special tangent path. For example, the energy, which always behaves like $\delta^{2}$ in mean-field theory, varies like $g^{4}$ on this path, yielding $\widetilde{\alpha}=-2$. Note that the results $\widetilde{z}=\widetilde{v}=1$ are consistent with the scaling prediction (3.22) for the behavior of $\widetilde{v} \widetilde{z}$ on the tangent trajectory.

\section{Generic transition}

Away from the multicritical point (i.e., for $g \neq 0$ ), the critical behavior of the Mott insulator to superfluid transition changes since, as we have just seen, $\lambda_{g}>0$, so $g$ is a relevant perturbation at the $(d+1)$-dimensional $X Y$ fixed point. This generic critical behavior can be inferred from a perturbative RG wherein $g$ is held fixed. The $\omega^{2} \psi^{2}$ operator is then irrelevant and can be ignored, and the 
action (4.3) reduces to the path-integral representation of a fluid of interacting bosons in the continuum. In terms of the original lattice model, this fluid corresponds (roughly) to the gas of excess quasiparticles (or holes) which are present when the density deviates slightly from commensurate (integer) values. The critical behavior of this model has been studied extensively. ${ }^{6-8}$ Naive power counting with the coefficients of $k^{2} \psi^{2}$ and $i \omega \psi^{2}$ held fixed implies that $z=2$, whereupon $u$ is irrelevant for $d>d_{c}=2$, in agreement with the value for $d_{c}$ deduced by scaling arguments in Sec. III. At the resulting (Gaussian) fixed point, $v=\beta=\frac{1}{2}$ and $\eta=0$, implying a Mott gap which vanishes linearly at the transition, $E_{g} \sim \delta^{z v} \sim \delta$. It is worth noting that the exponent equality in (3.22), $z v=1$, continues to hold for $d>d_{c}$, despite the fact that the hyperscaling expressions for $\kappa$ and $\kappa_{s}$ used in its derivation in Sec. III are violated. As usual, the $\psi^{4}$ operator is dangerously irrelevant, causing this breakdown of hyperscaling for $d>d_{c}$. For example, since $f_{s}(\delta) \sim-\delta^{2} / u$, the compressibility is nonsingular at the transition, in contrast to the hyperscaling prediction (3.18b).

For $d<2, u$ is relevant at the Gaussian fixed point and a perturbative $\mathrm{RG}$ in powers of $\varepsilon=2-d$ can be developed. ${ }^{7}$ Working from the insulating phase, $r \geq 0$, one finds that due to the special form of the propagator

$$
G_{0}^{-1}(k, \omega)=i \omega+k^{2}+r,
$$

the self-energy, $\Sigma=G_{0}^{-1}-G^{-1}$, vanishes at every order in $u$ (this is true for $d \geq 2$ as well). In each diagram, all of the poles in $\omega$ lie in the complex upper-half plane, so that the frequency integral can be deformed into the lowerhalf plane to give zero. The vanishing of $\Sigma$ is a reflection of the fact that the Mott phase has only virtual particle and hole excitations and thus a vanishing (real) excess density of bosons. By contrast, $u$ itself is renormalized. In fact, as shown by Uzunov, ${ }^{7}$ the only contributing diagrams form a geometric series, known as ladder diagrams, giving for the renormalized $u$ :

$$
u^{\prime}=b^{2-d} u /(1+a u) ; a=\frac{1}{2} \int_{k}^{\prime} \frac{1}{k^{2}},
$$

where the $k$ integration is over a momentum shell with $1 / b<k<1$, with rescaling parameter $b$. Equation (4.4) gives a new fixed point at $u^{*}=O(2-d)$ and $r=0$. Since $\Sigma=0$, this fixed point has Gaussian exponents $v=\frac{1}{2}, z=2$, and $\eta=0$. These exponent values presumably hold all the way down to the lower critical dimension $\left(d_{l}=1\right)$, since the RG is carried out to all orders in $u$. This implies that the Mott gap vanishes linearly in all dimensions $d$. In light of the phase diagram (Fig. 1) this result is not too surprising: As one approaches the phase boundary from the Mott phase, the gap (which is proportional to the change in $\mu$, at fixed $J$, required to reach the phase boundary) will clearly vanish linearly in $\delta$, except at the multicritical point.

\section{B. Bosons with disorder in one dimension}

We now consider the zero-temperature disordered boson problem in one space dimension. Since for $d=1$ and
$T=0$ the superfluid state has only algebraic or quasilong-range order, rather than true long-range order, transitions from the superfluid to insulating states can be accessed perturbatively in the strength of the external potential. Previous work on one-dimensional Bose systems has been restricted to bosons subjected either to periodic or to random potentials. To mimic the Bose glass to superfluid transition in the disordered lattice models of Secs. I-III, however, we must study a potential with both a periodic piece (the lattice), and a random piece (the disorder). Specifically, we investigate the transitions between the superfluid state, the Mott insulator, and the Bose glass in the context of the phase diagrams of Sec. II and the scaling arguments of Sec. III.

We consider explicitly a one-dimensional system of repulsively interacting bosons moving in a periodic potential, $u \cos (2 \pi x)$, plus a random potential $V(x)$, specified below. We employ a representation of onedimensional bosons due to Haldane, ${ }^{13}$ which expresses the relevant low-energy features of the Hamiltonian in terms of an operator $\Pi(x)$ which represents (small) deviations of the Bose density from its mean value (in the absence of the external potential). Following Giamarchi and Schulz, ${ }^{5}$ a convenient functional integral representation for the partition function can then be obtained by working in a basis of states diagonal in an operator $\widetilde{\theta}(x)$, defined as $\partial_{x} \widetilde{\theta}(x) \equiv \pi \Pi(x)$. This procedure is outlined in Appendix B. One thereby obtains the following form for the $T=0$ partition function:

$$
Z=\int D \widetilde{\theta}(x, \tau) \exp (-S),
$$

with $S=S_{O}+S_{P}+S_{R}$, and

$$
\begin{aligned}
S_{0}= & \frac{K}{2 \pi} \int d x d \tau\left[C_{2}^{-1}\left(\partial_{\tau} \widetilde{\theta}\right)^{2}+C_{2}\left(\partial_{x} \widetilde{\theta}\right)^{2}\right], \\
S_{P}= & -u \int_{x, \tau} \cos \left[2 \pi\left(\rho_{0}-1\right) x+2 \widetilde{\theta}(x, \tau)\right], \\
S_{R}= & -\int_{x, \tau} \delta \mu(x) \partial_{x} \widetilde{\theta}(x, \tau) \\
& +\int_{x, \tau}\left[\xi(x) e^{i 2 \widetilde{\theta}(x, \tau)}+\text { c. c. }\right] .
\end{aligned}
$$

Here, $S_{0}$ describes the second sound mode of the superfluid phase in the absence of an external potential, with second sound velocity $C_{2}=\left(\rho_{s} / m \kappa\right)^{1 / 2}$, and $S_{P}$ and $S_{R}$ represent the contributions from the periodic and random potentials, respectively. When $S_{P}=S_{R}=0$, superfluid correlations decay as a power law,

$$
\left\langle\hat{\boldsymbol{\Phi}}(x) \hat{\boldsymbol{\Phi}}^{+}(0)\right\rangle \sim|x|^{-K / 2},
$$

with an exponent $K$. This parameter is related directly to $\rho_{s}$ and the compressibility

$$
(\pi K)^{2} \equiv m / \rho_{s} \kappa
$$

In arriving at (4.8) it was assumed that the Bose density $\rho_{0}$ was nearly commensurate with the periodic potential, $\rho_{0} \simeq 1$. In (4.9), $-\delta \mu(x)$ and $\xi(x)$ are the contributions of the random potential $V(x)$ with fourier components near $k=0$ and $k= \pm 2 \pi \rho_{0}$, respectively. For convenience, these can be taken as satisfying a Gaussian white noise distribution, 


$$
\begin{aligned}
& \overline{\delta \mu(x)}=0, \overline{\delta \mu(x) \delta \mu\left(x^{\prime}\right)}=\Delta \delta\left(x-x^{\prime}\right), \\
& \overline{\xi(x)}=0, \quad \overline{\xi^{*}(x) \xi\left(x^{\prime}\right)}=D \delta\left(x-x^{\prime}\right) .
\end{aligned}
$$

The constraint that the mean density be $\rho_{0}$ fixes the boundary conditions on $\widetilde{\theta}(x, \tau)$ in (4.6). Specifically, we require that for a system of length $L$,

$$
L^{-1}[\widetilde{\theta}(L, \tau)-\widetilde{\theta}(0, \tau)] \rightarrow 0
$$

as $L \rightarrow \infty$. If $\widetilde{\theta}(x, \tau)$ is viewed as representing the "height" of a two-dimensional interface, this is equivalent to requiring that the interface be "flat."

Although there is no lattice in the above model, a rough correspondence with the lattice models studied in Secs. II and III can be made through the association of the bandwidth for a particle with mass $m$ in the potential $u \cos (2 \pi x)$ with the lattice hopping strength $J$. Thus, e.g., in the large $u$ limit one has, $J \sim \exp \left[-(u m)^{1 / 2}\right]$, so that increasing $K$ or $u$ corresponds to decreasing $J$.

\section{Pure periodic potential}

In the absence of any disorder $\left(S_{R}=0\right)$, and for a density commensurate with the periodic potential $\left(\rho_{0}=1\right), S$ reduces to the Sine-Gordon model. Standard RG analysis $^{32}$ shows that the Gaussian fixed line, $u=0$, is stable with respect to small $u$ for $K$ smaller than a critical value, $K_{M}=\frac{1}{2}$. For $K>K_{M}$, the potential $u$ is relevant, and the superfluid state (at $u=0$ ) is unstable with respect to a locked insulating state. The insulating state is in fact an incompressible Mott state, with the concomitant Mott gap, as described in Sec. II (Fig. 1). This becomes apparent if one thinks of $\widetilde{\theta}(x, \tau)$ as an interfacial "height." The insulating phase corresponds to a smooth (as opposed to rough) interface which has a nonzero free energy, $f_{s}$, for the creation of steps. An applied chemical potential field, $-\mu \int_{x, \tau}\left(\partial_{x} \widetilde{\theta}\right)$, tends to tilt the interface by putting in steps, which corresponds to adding particles. Clearly one needs $|\mu|>f_{s}$ before steps are introduced or, equivalently, before the boson density deviates from $\rho_{0}=1$. Thus, for $\mu=0$, one has an incompressible Mott insulator with $\partial \rho / \partial \mu=0$, and a nonvanishing Mott gap, $E_{g}=2 f_{s}$. At the (Kosterlitz-Thouless) ${ }^{33}$ roughening transition $^{34}$ the step free energy vanishes as $f_{s} \sim \xi^{-1}$, where $\xi$ is the correlation length exponent for the twodimensional $X Y$ model. Thus, as the superfluid transition is approached from the insulating side (by decreasing $K$ or $u$ at fixed density $\left.\rho_{0}=1\right)$ the Mott gap vanishes as ${ }^{33}$ $E_{g} \sim \exp \left(-1 / \sqrt{K-K_{c}}\right)$, where $K_{c}$ is the critical value of $K$, for fixed positive $u$. This is entirely consistent with the results deduced in Sec. IV A, where, when $J$ was increased at fixed commensurate density, $E_{g}$ vanished with the $(d+1)$-dimensional $X Y$ correlation length exponent.

For densities away from commensurability $\left(\rho_{0} \neq 1\right)$ the superfluid (rough) phase is always stable. Indeed, even for large $u$, the boundary conditions on $\widetilde{\theta}$ require configurations which have steps [with step density $\left.\left(\rho_{0}-1\right)\right]$ which are then free to meander and lead to a rough phase. These steps correspond to excess bosons ("on top of" the commensurate insulator) which are able to condense into a superfluid state. Thus the physics is completely consistent with our phase diagram (Fig. 1) for lattice bosons without disorder: It is clear from that figure that at any density for which the number of particles per site is not an integer, the system is superfluid all the way down to $J=0$. In the presence of longer range interactions, higher-order commensurate Mott phases are possible which break the spatial symmetry; we will not consider these here.

\section{Quenched random potential}

In the presence of the (quenched) random potential, (4.9), the ensemble-averaged free energy, $F=-(1 / \beta) \overline{\ln Z}$, can be (formally) evaluated through use of the replica trick: $\ln Z \rightarrow\left(Z^{n}-1\right) / n$, as $n \rightarrow 0$. For Gaussian distributions of $\delta \mu(x)$ and $\xi(x)$ [(4.11)-(4.12)], the random average can be performed exactly, yielding a "replicated" action $\widetilde{S}$ :

$$
\overline{Z^{n}}=\int \prod_{\alpha} D \widetilde{\theta}_{\alpha}(x, \tau) e^{-\tilde{S}},
$$

where $\alpha=1, \ldots, n$ labels the replicas. For a potential with no periodic component $(u=0)$ as considered here, one can absorb the $\delta \mu(x)$ term of (4.9) into $S_{0}$ by the transformation

$$
\widetilde{\theta} \rightarrow \widetilde{\theta}+\frac{\pi}{C_{2} K} \int_{-\infty}^{x} \delta \mu\left(x^{\prime}\right) d x^{\prime} \equiv \widetilde{\theta}+w(x) / 2 .
$$

This variable change simply produces a shift in the phase of the complex random potential $\xi(x)$, which can be absorbed by a redefinition of $\xi$. One thereby obtains a "replicated" action $\widetilde{S}=\widetilde{S}_{0}+\widetilde{S}_{R}$ with

$$
\begin{aligned}
& \widetilde{S}_{0}=\sum_{\alpha} \frac{K}{2 \pi} \int_{k, \omega}\left(C_{2} k^{2}+C_{2}^{-1} \omega^{2}\right)\left|\widetilde{\theta}_{\alpha}(k, \omega)\right|^{2}, \\
& \widetilde{S}_{R}=-D \int_{x, \tau_{1}, \tau_{2}} \sum_{\alpha, \beta} \cos \left[2 \widetilde{\theta}_{\alpha}\left(x, \tau_{1}\right)-2 \widetilde{\theta}_{\beta}\left(x, \tau_{2}\right)\right] .
\end{aligned}
$$

The Bose glass to superfluid transition can now be investigated by studying the stability of the fixed line through perturbative RG analysis for small $D$, as demonstrated by Giamarchi and Schulz. ${ }^{5}$ We briefly summarize their calculations. First rescale time so that $C_{2}=1$ in (4.15). Then integrate out $\widetilde{\theta}(k, \omega)$ with $\Lambda / b<k<\Lambda$ and rescale as $k^{\prime}=b k$ and $\omega^{\prime}=b^{z} \omega$ to complete the RG transformation. The parameter $z$ will be chosen so that the coefficients of $k^{2}\left(\widetilde{\theta}_{\alpha}\right)^{2}$ and $\omega^{2}\left(\widetilde{\theta}_{\alpha}\right)^{2}$ remain invariant under the RG transformation, (i.e., that the sound speed is unrenormalized). Working to lowest order in $D$ and $3-2 / K$, one thereby obtains the $\mathrm{RG}$ recursion relations $(l=\ln b)$ :

$$
\begin{aligned}
& \frac{\partial D}{\partial l}=(3-2 / K) D, \\
& \frac{\partial K}{\partial l}=D / 2,
\end{aligned}
$$

with

$$
z=1+D / 2 K \text {. }
$$

These equations have a stable fixed line at $D=0$ for all $K$ less than a critical value $K_{G}=\frac{2}{3}$. Thus, at least for 
small $D$ where the recursion relations are valid, the RG flows for $K<K_{G}$ terminate in the fixed line; i.e., as in the pure case, the system is a superfluid, with algebraicallydecaying correlations for $K$ sufficiently small. For $K>K_{C}$, on the other hand, the flows run off to large values of $D$ beyond the range of validity of our perturbative equations. This runaway to the strong-disorder limit was interpreted by Giamarchi and Schulz as the signature of a localized insulator, consistent with our'expectations of a Bose glass phase occurring for sufficiently weak hopping (i.e., large $K$ ). The positive compressibility of this state (which differentiates it from the Mott insulating phase) can be established from the action (4.15) and (4.16) as follows: For each configuration $\widetilde{\theta}_{\alpha}(x, \tau)$ transform to a tilted configuration, $\widetilde{\theta}_{\alpha} \rightarrow \widetilde{\theta}_{\alpha}+\phi x$, with constant tilt angle $\phi$. Since $\widetilde{S}_{2}$ is invariant under this transformation, only $\widetilde{S}_{0}$ is modified. However, $\widetilde{S}_{0}$ changes only quadratically with $\phi$, so that the free energy for producing a small "tilt," vanishes more rapidly than linearly; in roughening language the step free energy is zero. This implies (in contrast to the situation for the pure periodic potential), a nonzero compressibility, characteristic of a disorderdominated Bose glass phase.

The Bose glass to superfluid transition described by (4.17) has, as demonstrated by Giarmarchi and Schulz, ${ }^{5}$ critical properties different from the Kosterlitz-Thouless transition which describes the Mott to superfluid transition in the pure case. For example, the universal jump in the stiffness (or exponent) $K$ at the two transitions is different since $K_{G} \neq K_{M}$. Moreover, since $K$ is renormalized at first order in $D$, the superfluid correlation length in the Bose glass diverges as $\xi \sim \exp \left[-\left(K-K_{c}\right)^{-1}\right]$ near the superfluid critical point, in contrast to the Kosterlitz-Thouless form $\exp \left[-\left(K-K_{c}\right)^{-1 / 2}\right]$ in the Mott state (see the end of this section for the implications of this).

The Bose glass-superfluid critical behavior provides a nontrivial confirmation of the scaling arguments advanced in Sec. III. Firstly, since the critical fixed point occurs at $D=0$, we obtain immediately from (4.18) that $z=1$, in agreement with our scaling prediction $z=d$. Moreover, from (3.8), since $z=d=1$, superfluid correlations at criticality decays as $|x|^{-\eta}$ implying $\eta=K_{G} \equiv \frac{2}{3}$. This value of $\eta$ satisfies the exponent inequality, obtained in Sec. III from scaling; $\eta \leq 2-d=1$.

To confirm that the compressibility is finite at the transition requires more work, since the scaling expression (3.18b) $\kappa \sim \delta^{v(d-z)}$ is ambiguous when $z=d=1$ and $v=\infty$. Since $K$ and $C_{2}$ together determine uniquely $\rho_{s}$ and $\kappa$ [see (4.10)], it is easier to establish that $\kappa(\delta=0)$ is finite by deriving new $\mathrm{RG}$ recursion relations with $z \equiv 1$, allowing both $K$ and $C_{2}$ to renormalize. One finds that (4.17a) is unchanged, whereas

$$
\begin{aligned}
& \frac{\partial K}{\partial l}=\frac{C_{2} D}{2}, \\
& \frac{\partial C_{2}}{\partial l}=\frac{-D C_{2}^{2}}{2 K} .
\end{aligned}
$$

These recursion relations, together with $(4.17 \mathrm{a})$, have a fixed point at $K=K_{G}, D=0$. To establish that $\kappa(\delta=0)$ is finite, one must verify that flows near $K=K_{G}, D=0$ are consistent with a nonzero fixed-point value of $C_{2}$, denoted $C_{2}^{*}$. Putting $C_{2}=C_{2}^{*}$ in (4.19a) implies a critical separtrix flowing into $K=K_{G}, D=0$, along which $D(l)$ vanishes algebraically, $D(l) \sim 1 / l^{2}$ for $l \rightarrow \infty$. This decay is fast enough that integration in $(4.19 \mathrm{~b})$ with $K=K_{G}$ gives a nonzero fixed point value for $C_{2}(l=\infty)=C_{2}^{*}$, consistent with the assumption. Thus we have established that at criticality both $K$ and $C_{2}$ are finite and nonzero, implying the same, in turn, for $\rho_{s}$ and $\kappa$.

\section{Periodic plus random potential}

We now consider the effect of turning on a small periodic potential, $u>0$, in (4.8), choosing the commensurate density $\rho_{0}=1$. Performing the same shift of $\widetilde{\theta}$ described in (4.14), one arrives at the replicated action, $\widetilde{S}=\widetilde{S}_{0}+\widetilde{S}_{P}+\widetilde{S}_{R}$ with $\widetilde{S}_{0}$ and $\widetilde{S}_{R}$ given in (4.15) and (4.16) and

$$
\widetilde{S}_{P}=-u \int_{x, \tau} \sum_{\alpha} \cos \left[2 \widetilde{\theta}_{\alpha}+w(x)\right]
$$

The average over $w(x)$, defined in (4.14), must be carried out perturbatively in powers of $u$. Since $w(x)$ describes a random walk in $x$, the term linear in $u$ vanishes. The quadratic term in $u$ has the form

$$
\begin{aligned}
& \widetilde{S}_{P}=-\frac{u^{2}}{4} \int_{x_{1} \tau_{1}} \int_{x_{2} \tau_{2}} \sum_{\alpha \beta} \cos \left[2 \widetilde{\theta}_{\alpha}\left(x_{1}, \tau_{1}\right)-2 \widetilde{\theta}_{\beta}\left(x_{2}, \tau_{2}\right)\right] f\left(x_{1}-x_{2}\right) \\
& f(x)=\exp \left[-\Delta\left(2 \pi / K C_{2}\right)^{2}|x|\right]
\end{aligned}
$$

The disorder manifests itself only on sufficiently large length scales:

$$
\left|x_{1}-x_{2}\right|>\left(K C_{2}\right)^{2} / \Delta \text {. }
$$

For

$$
\left|x_{1}-x_{2}\right| \ll<\left(K C_{2}\right)^{2} / \Delta,
$$

$\Delta$ might just as well be zero, and the system experiences only the periodic part of (4.20). Anticipating that only the dominant long-wavelength part of Eq. (4.21) will be relevant near the onset of superfluidity or in the superfluid state, we Taylor expand $\widetilde{\theta}\left(x_{2}, \tau_{2}\right)$ about $\left(x_{1}, \tau_{2}\right)$, keeping only the leading term in powers of $x_{2}-x_{1}$, which is identical in structure to (4.16), and has coefficient $\left(u K C_{2} / 2 \pi\right)^{2} / 2 \Delta$. The resulting action is identical to that of the Giamarchi-Schulz ${ }^{5}$ problem reviewed above, the effect of the periodicity having been subsumed in a simple renormalization of $D$ in (4.16): 


$$
D \rightarrow \widetilde{D}=D+\left(u K C_{2} / 2 \pi\right)^{2} / 2 \Delta .
$$

Since the perturbative methods of Giamarchi and Schulz ${ }^{5}$ apply only for weak coupling, $\widetilde{D} \ll 1$, we can only treat the situation where both $D$ and $\left(u K C_{2}\right)^{2} / \Delta$ are small. In this portion of parameter space, however, we can immediately infer the existence of a superfluid phase for $K<K_{G}$, controlled by the Gaussian fixed line at $\widetilde{D}=0$. For $K>K_{G}$, and RG flows run off, as we have seen, to large $\widetilde{D}$, signalling the occurrence of an insulating phase. The transition between these two states is in the universality class of the Giamarchi-Schulz problem, which strongly suggests that the insulating phase is a Bose glass. We cannot, however, directly verify this conjecture by analyzing the RG trajectories, since they rapidly flow out of the range of validity of perturbation theory. Assuming that the flow is to the Bose glass phase, one concludes that commensurate periodic potentials weak compared to the disorder $\left(u^{2}<<\Delta\right)$ do not affect in any serious way the phases and phase transitions of one-dimensional bosons at $T=0$. This is consistent with the arguments of Sec. II, where we concluded that sufficiently strong disorder eliminated the Mott insulating phase, leaving only the Bose glass and superfluid states. It is clear that weak incommensurate potentials $\left(\rho_{0} \neq 1\right)$ will be even less effective in altering the phase diagram, so we hypothesize that any weak periodic potential plays a negligible role. For large $u$ and weak disorder, we expect a more complicated phase diagram where the Mott phase can occur, as in Sec. II and Fig. 2.

\section{Bosons with disorder in dimension $d>1$}

We have just seen how, in one-dimension, explicit RG calculation of the Bose glass-superfluid critical behavior confirms the prediction for $z$ and bound on $\eta$ from Sec. III and allows the computation of $v$ and the rest of the critical exponents. Analogous results for $d>1$ - even limited to an $\varepsilon$ expansion about some upper critical dimension-would be highly desirable. The $\varepsilon$ expansion can indeed be formally generalized to yield consistent recursion relations for the dirty boson problem, but various conceptual and technical difficulties leave one uneasy about the validity of the calculation. Here we only outline the approach. Details will appear elsewhere. ${ }^{8}$

The calculations leading to Eqs. (4.2) and (4.3) for the pure problem can be straightforwardly repeated for the random boson problem (2.1) with nearest-neighbor hopping and a random potential. One obtains the classical action

$$
\begin{aligned}
S^{\prime}= & \frac{1}{2} \int_{k, \omega}\left(k^{2}+\omega^{2}\right)|\psi(k, \omega)|^{2}+\frac{1}{2} \sum_{i} \widetilde{r}_{i}\left|\psi_{i}(\tau)\right|^{2} \\
& +g^{\prime} \int_{k, \omega} i \omega|\psi|^{2}+u \int_{x, \tau}|\psi|^{4},
\end{aligned}
$$

where $2 \widetilde{r}_{i} \equiv J^{-1}-A_{i}$ with $A_{i}$, defined in Appendix A Eq. (A7b), a random function of $i$ related to the local random variations of the chemical potential, $\delta \mu_{i}$. The coefficients of all the other terms in (4.23) are also, strictly speaking, random functions of position. As usual, however, only the randomness in the $k$-and $\omega$-independent part of the coefficient of $|\psi|^{2}$ (i.e., in $\widetilde{r}_{i}$ ), is relevant for computing critical properties; all other coefficients can be replaced by their mean values. Following the standard prescription for computing critical properties in random systems, we write $\widetilde{r}_{i}=\left(r+\delta r_{i}\right)$, with $r$ the mean of $\widetilde{r}_{i}$, and $\delta r_{i}$ a random variable with zero mean. Of course this prescription works only when $r$ is finite; this requires both denominators in (A7b) to be bounded below, i.e., that at $J=0$ the number of bosons, $n_{i}$, is the same for each site, $i$, and $n_{i}$ satisfies

$$
\left(\mu+\delta \mu_{i}\right) / V<n_{i}<1+\left(\mu+\delta \mu_{i}\right) / V .
$$

These conditions hold only for bounded distributions, such as (2.2a) with $\Delta<V / 2$, and for values of $\mu$ for which there is a Mott insulating (MI) state (Fig. 2).

If, as argued in Sec. II, the phase diagram in the presence of bounded disorder is like that in Fig. 2(a), then the onset transition to superfluidity can only occur from the Bose glass state. One might then hope that the action (4.23) can be successfully used to study the critical behavior of this onset transition. The most natural procedure would be to first evaluate $S^{\prime}$ in a saddle point approximation [with $\psi(x, \tau)$ a constant] plus Gaussian fluctuations (0-loop), and then develop a systematic loop expansion in powers of $u$ and the strength of the disorder, $w \equiv\left\langle\delta r^{2}\right\rangle$. While this approach can indeed be carried forth, technical problems arise as to how to treat the frequency sums (see below).

To proceed, it is convenient to use the replica trick to average over the randomness. Keeping only the lowestorder (i.e., the second) moment, $w$, of the $\delta r_{i}$ distribution, one obtains the replicated action

$$
\begin{gathered}
S^{\prime \prime}=\frac{1}{2} \sum_{\alpha=1}^{N}\left[\int_{k, \omega}\left(k^{2}+\omega^{2}+r\right)\left|\psi_{\alpha}(k, \omega)\right|^{2}\right. \\
\left.\quad+2 g^{\prime} \int_{k, \omega} i \omega\left|\psi_{\alpha}\right|^{2}+2 u \int_{x, \tau}\left|\psi_{\alpha}\right|^{4}\right] \\
-w \int_{x, \tau, \tau^{\prime}} \sum_{\alpha, \beta=1}^{N}\left|\psi_{\alpha}(x, \tau)\right|^{2}\left|\psi_{\beta}\left(x, \tau^{\prime}\right)\right|^{2}
\end{gathered}
$$

As usual, higher moments of the distribution give rise to irrelevant operators, and the number, $N$, of replicas must be set to zero at the end of the calculation. Since the random variables, $\delta r_{i}$, are purely static, the replica-mixing term [with coefficient $w$ in (4.24)] is nonlocal in time. Such nonlocality is a familiar consequence of quenched disorder in quantum problems at $T=0 .{ }^{4}$

One can now try to compute the critical properties of the action (4.24) via $\varepsilon$-expansion methods. As in the pure case, the generic situation is that $g$, the coefficient of $i \omega|\psi|^{2}$, is nonzero. The $\omega^{2}|\psi|^{2}$ term is therefore irrelevant and can be dropped. Treating the nonlocal $w$ term in (4.24) by the standard technique ${ }^{35}$ of imagining that it is nonlocal in $\varepsilon_{\tau}$ dimensions and local in the other $d$, one readily generates formal $R G$ recursion relations in a double power series expansion in $\varepsilon_{\tau}$ and $\varepsilon \equiv 4-d-\varepsilon_{\tau}$. (The physically-interesting cases are of course $\varepsilon=0$ or 1 , with $\varepsilon_{\tau}=1$.) Unfortunately, the $i \omega$ form of the frequency dependence of the propagator, which rendered the generic Mott insulator-superfluid transition trivial in the pure 
case (Sec. IV A), makes the random problem intractable as posed: One finds that the recursion relation for $w$ is simply

$$
\frac{\partial w}{\partial l}=\left(\varepsilon+\varepsilon_{\tau}\right) w+B w^{2}+, \ldots,
$$

where $B$ is a positive coefficient. Since, as the second moment of a random distribution, $w$ starts out positive, it flows under RG iteration to large values where perturbation theory is not valid. Thus the recursion relations as they stand have no stable, accessible fixed point, and provide no information about the Bose glass to superfluid transition. This is consistent with the possibility discussed earlier that the upper critical dimension is infinite and $z=d$ for all $d$. However, if a (physically unnatural) high-frequency cutoff, $\omega_{\Lambda}$, is imposed on the frequency integrals, a nontrivial $O\left(\varepsilon, \varepsilon_{\tau}\right)$ fixed point can be found. ${ }^{36}$ Above $d=4$, in contrast to more standard cases, both the random and Gaussian fixed points are stable with distinct domains of attraction, ${ }^{37}$ so that for weak disorder the transition is mean field with Gaussian exponents and for strong disorder is likely to be the random fixed point with $z=d$. As $d$ decreases towards four, the domain of attraction of the Gaussian fixed point shrinks to zero, with only the random fixed point stable for $d<4$. Such an unusual scenario avoids the pitfalls of a standard mean-field onset, mentioned in Sec. III.

It should be emphasized that the above perturbative renormalization-group scheme is, as usual, an expansion about mean-field theory. In the mean-field limit, however, the Bose glass phase does not occur at all, so that the transition to superfluidity occurs directly from the Mott insulator. (One can see the absence of the Bose glass phase in mean-field theory from the exact solution of the infinite-range hopping model in II B.) This suggests that because of nonperturbative effects the renormalizationgroup expansion may not be valid for studying the onset of superfluidity from the Bose glass phase, which would be consistent with the conclusion that $d_{c}=\infty$ and $z=d$ for all $d$. While we have no definitive argument in support of this conclusion, if further study does not resolve the conceptual and technical difficulties associated with the perturbative renormalization-group treatment, then we would conclude that the $z=d$ scenario is indeed the correct one.

In the (unlikely) event that the phase diagram is as in Fig. 2(c), with a direct Mott insulator to superfluid transition possible, then right at the tips of the Mott states (i.e., when the boson density is commensurate even in the superfluid state), arguments essentially identical to those of Sec. IV A show that the coefficient $g^{\prime}$ must vanish. The action (4.24) then reduces to a $d+1$-dimensional $X Y$ model with random impurities correlated in the time direction, a model studied in detail by Dorogovtsev. ${ }^{35}$ Having inadvertently dropped the $i \omega \psi^{2}$ term in their derivation, MHL (Ref. 4) argued that model (4.24) with $g^{\prime}=0$ should describe the Bose glass to superfluid transition. We believe (further arguments being given below) that this is incorrect. If, however, the phase diagram depicted in Fig. 2(c) is possible, then the special commensu- rate transitions would presumably be described by the MHL theory.

In the case of Fig. 2(b), the only direct Mott insulatorsuperfluid transitions occur at the tips, so it is tempting to hypothesize that the MHL theory would likewise apply to these transitions. Since the Bose glass states in Fig. 2(b) extend right up to the tips, however, it could well be that a more complicated multicritical fixed point describes the transition in this case. Since expressions like (4.24) do not easily allow for a description of both the Mott and Bose glass insulators, it is difficult to say anything definitive about this possibility. Furthermore, as we have argued in Sec. II, we believe that both phase diagrams shown in Figs. 2(b) and 2(c) are unlikely to obtain.

Finally, we note that the one-dimensional case provides strong evidence that a $(d+1)$-dimensional $X Y$ model with random impurities correlated in time, as considered by MHL, does not correctly describe the Bose glass to superfluid transition. Recall that even correlated impurities are strongly irrelevant about the Kosterlitz-Thouless fixed point in $(1+1)$ dimensions. Thus, the fact that the Bose glass-superfluid transition for $d=1$ is not of the Kosterlitz-Thouless type (Sec. IV B) strongly suggests that MHL's model is not relevant to this transition.

\section{EXPERIMENTAL RAMIFICATIONS AND CONCLUSIONS}

The models investigated in this paper have a natural experimental realization in systems wherein ${ }^{4} \mathrm{He}$ is confined in restricted geometries (such as Vycor, or other porous media), ${ }^{15,16}$ or to the surface of $2 \mathrm{D}$ substrates. ${ }^{38}$ In either case, the temperature, $T_{c}$, of the normalsuperfluid transition is observed to decrease with the density, $\rho$, of ${ }^{4} \mathrm{He}$ present, apparently extrapolating to zero at a positive value, $\rho_{c}(T=0)$, of $\rho$ (Fig. 5). Such systems therefore undergo superfluid onset transitions as $\rho$ increases at $T=0$ - precisely the kind of transition considered here. While the experimental feasibility of probing low-temperature behavior stops short of $T=0$, the transition to superfluidity at sufficiently low temperatures

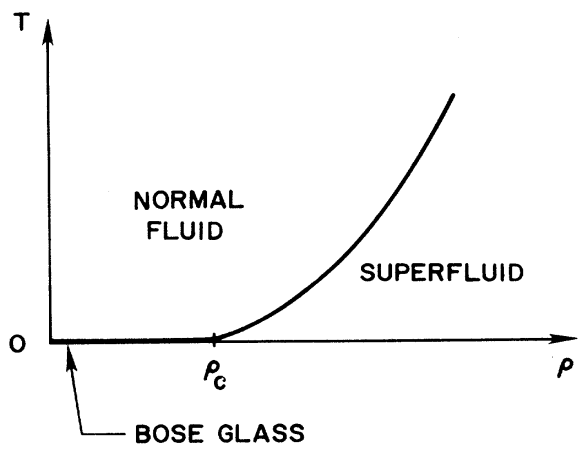

FIG. 5. Schematic phase diagram for ${ }^{4} \mathrm{He}$ confined in disordered restricted geometries, such as porous media. The zerotemperature superfluid-insulator transition crosses over to a line of conventional $\lambda$ transitions at $T>0$. Viewing this crossover as a standard multicritical-to-critical crossover enables explicit verifiable predictions to be made for $T$ small and $\rho$ near $\rho_{c}$. 
will be dominated by the $T=0$ fixed point until one gets very near to the critical density, $\rho_{c}(T)$. The crossover from the multicritical point at $T=0$ to the conventional critical line of $\lambda$ transitions at $T>0$ (Fig. 5), can be treated, as we see below, as a standard multicritical-to-critical crossover.

Before turning to specific experimental predictions, a word of caution is necessary. Throughout the paper we have ignored the possibility of attractive van der Waals interactions between the bosons which clearly are present in real ${ }^{4} \mathrm{He}$ systems. In the absence of disorder and at zero temperature, these attractive interactions can stabilize a self-bound superfluid liquid phase in coexistence with a zero density vapor. If such phase separation is present experimentally and the superfluid onset transition is first order, the theory developed in the preceding sections would be inapplicable. Such a situation might be the case for superfluid onset on extremely clean substrates. In the presence of disorder which is large compared with the magnitude of the attractive van der Waals interaction, however, phase separation will be destroyed and the transition to superfluidity at both zero and finite temperatures is likely to be continuous. In this case, the zero-temperature onset transition should be in the universality class of the Bose glass-superfluid transition discussed in Secs. II, IV B, and IV C. For ${ }^{4} \mathrm{He}$ absorbed in porous media, the transitions to superfluidity seem clearly continuous; ${ }^{15,16}$ the van der Waals interaction is thus probably unimportant for the critical behavior of the superfluid transition.

The scaling analysis of Sec. III yields specific predictions for experimental observables in the vicinity of the superfluid onset at $T=0$. We now derive expressions for several of the most accessible of these in terms of the distance, $\delta$, from the critical point at $T=0$ (as defined in Sec. III). As discussed in Sec. III, the effect of nonzero temperature in the path integral formulation of quantum mechanisms is to restrict the imaginary time to lie between 0 and $\beta \equiv 1 / k_{B} T$. This makes the system, viewed as a $(d+1)$-dimensional system, finite in the time direction, allowing the crossover to the positive temperature critical behavior to be deduced from finite-size scaling. The singular part, $f_{s}$, of the free energy density can thus be written [see (3.6)]

$$
\begin{aligned}
f_{s}(\delta, T) & \sim|\delta|^{v(d+z)} \widetilde{f}(T / \Omega) \\
& \sim|\delta|^{v(d+z)} \widetilde{f}\left(T|\delta|^{-z v}\right),
\end{aligned}
$$

in units where $k_{B}=\hbar=1$. This expresses the scaling of $\beta$, the system "size" in the time direction, in units of $\Omega^{-1}$ the unique characteristic time in the problem. The scaling function $\widetilde{f}(u)$, which approaches a constant [see (3.6)] as $u \rightarrow 0$, must have a singularity at some finite value, $u_{c}$, of its argument, corresponding to the critical line of $\lambda$ transitions at $T>0$ in Fig. 5. Thus the critical temperature, $T_{c}$, vanishes like

$$
T_{c}=u_{c} \delta^{z v}
$$

for small $\delta$. Since the argument of the scaling function above is just $u_{c} T / T_{c}$, the form (5.1) implies an asymptot- ically scale invariant shape for appropriately scaled temperature profiles of all the physical quantities discussed below. In the pure case, because of the presence of the dangerous irrelevant operator $u \phi^{4}$ for $d>2$, this is manifestly false. ${ }^{8}$ However, in the random case, provided hyperscaling holds, sufficiently close to onset we do expect $(5.1)$ to be valid.

The superfluid density, the primary quantity in experiments on ${ }^{4} \mathrm{He}$ in confined geometries, obeys [from (3.18a)] a scaling relation similar to $(5.1)$ :

$$
\rho_{s} \sim \delta^{v(d+z-2)} \widetilde{\rho}_{s}\left(T \delta^{-z v}\right) .
$$

Likewise the total density $\rho$, being simply the derivative with respect to $\delta$ (i.e., $\mu$ ) of $f$, has a singular part which behaves like

$$
\left(\rho-\rho_{c}\right)_{s} \sim|\delta|^{v(d+z)-1} \widetilde{\rho}\left(T|\delta|^{-z v}\right) .
$$

Note, however, that if $v(d+z)-1 \geq 1$ (i.e., if $\kappa \sim \partial \rho / \partial \delta$ remains finite at the onset transition [see (3.12)]), the behavior of $\rho$ near $\rho_{c}$ will be dominated by the piece analytic in $\delta$, so that for $\delta \rightarrow 0$,

$$
\rho-\rho_{c} \sim \delta \text {. }
$$

This is important, since it permits the variable $\delta$ to be replaced by the experimentally measurable quantity $\rho-\rho_{c}$ in the relations derived in this section, thereby rendering feasible direct comparison with experiment. Since, as discussed in Sec. III, (3.24), valid for any random system, implies $v(d+z) \geq 2$ for any $z \geq 0$, we assume henceforth that $(5.4 \mathrm{~b})$, rather than $(5.4 \mathrm{a})$, describes the asymptotic behavior of $\rho-\rho_{c}$.

The velocity with which fluctuations in the phase of the superfluid order parameter propagate (i.e., the second sound velocity, $C_{2}$ ) is given, according to (3.13) and (3.18), by

$$
C_{2}=\left(\rho_{s} / \kappa\right)^{1 / 2} \sim \delta^{v(z-1)} \widetilde{C}_{2}\left(T \delta^{-z v}\right) .
$$

Another important quantity is the particle conductivity, $\sigma$, which follows from the current response under the action of an infinitesimal externally-imposed chemical potential gradient. In the superfluid phase, the frequency dependent conductivity $\sigma(\omega)$ varies as $\sigma(\omega) \sim \rho_{s} / \omega$. Combining the scaling of $\rho_{s}$ in (3.18) with the fact that frequencies scale with $\delta^{z v}$ gives an $\omega=0$ conductivity of the form

$$
\sigma \sim|\delta|^{v(d-2)} \widetilde{\sigma}\left(T|\delta|^{-z v}\right)
$$

In the Bose glass phase we expect the conductivity, as for Fermi glasses, to be controlled by variable-range hopping at low temperatures: $\sigma(T) \sim \exp \left[-\left(T_{H} / T\right)^{y}\right]$, where $y<1$ and, from (5.6), $T_{H} \sim|\delta|^{z v}$. The scaling expression (5.6), which can alternatively be cast as $\sigma \sim T^{(d-2) / z} \Sigma\left(T|\delta|^{-z v}\right)$, differs slightly from that given in Eq. (8) of Ref. 3. Although the two forms agree when $z=d$, we believe (5.6) is generally the correct one. The argument in Ref. 3, which uses scaling of the particle diffusivity $D(\equiv \sigma / \kappa)$, is, we now believe, incorrect, since at $T=0$ bosons do not diffuse.

Finally, the superfluid coherence length $\xi$ behaves like 


$$
\xi \sim|\delta|^{-v} \widetilde{\xi}\left(T|\delta|^{-z v}\right)
$$

The scaling functions $\widetilde{\rho}_{s}(u), \widetilde{\rho}(u), \widetilde{C}_{2}(u), \widetilde{\sigma}(u)$, and $\widetilde{\xi}(u)$ all approach constants as $u \rightarrow 0$, thereby reducing to their correct zero-temperature limits, and are singular at $u=u_{c}$ with singularities appropriate to the finitetemperature $\lambda$ transition. Since the specific-heat exponent, $\alpha_{T}$, at the $\lambda$ transition is negative, Harris' criterion $^{39}$ implies that the disorder is irrelevant so that the positive temperature critical behavior is that of the pure system. There will, however, be very slow transients associated with $\alpha_{T}$ being very close to zero.

The scaling functions $\widetilde{\rho}_{s}$ and $\widetilde{C}_{2}$, which characterize properties unique to the superfluid, must vanish for $\delta<0$ or $u>u_{c}$. On the other hand, $\widetilde{\rho}(u), \widetilde{\sigma}(u)$, and $\widetilde{\xi}(u)$ behave like $u^{[v(d+z)-1] / z v}, u^{(d-2) / z}$, and $u^{-1 / z}$, respectively as $u \rightarrow \infty$ (i.e., as $\delta \rightarrow 0$ for fixed $T$ ), implying the vanishing or divergence like $T^{[v(d+z)-1] / z v}, T^{(d-2) / z}$, and $T^{-1 / z}$ of $\left(\rho-\rho_{c}\right)_{s}, \sigma$, and $\xi$, respectively, as $T \rightarrow 0$ for $\delta=0$. Note that the above scaling forms, (5.2) and (5.5)-(5.7) predict that if each quantity is scaled by their zero-temperature values and temperature is scaled by $T_{c}$, then the resulting temperature profiles, at fixed $\delta$, approach universal functions for $\delta$ small.

Eliminating $\delta$ from the scaling forms (5.1)-(5.7), one can derive relations for quantities which are directly accessible experimentally. For example, from (5.2)-(5.5) follow various connections between the critical temperature and the $T=0$ limits of $\rho_{s}, C_{4}$, and $\delta \rho$ :

$$
\begin{aligned}
& T_{c} \sim\left[\rho_{s}(0)\right]^{x}, \quad x \equiv z /(d+z-2) ; \\
& T_{c} \sim\left[C_{2}(0)\right]^{y}, \quad y \equiv z /(z-1) ; \\
& \rho_{s}(0) \sim\left(\rho-\rho_{c}\right)^{\xi}, \quad \zeta \equiv v(d+z-2) .
\end{aligned}
$$

Equations (5.8) and (5.9) are particularly useful, 'in that the exponents $x$ and $y$ do not involve $v$, but depend only on the dynamic exponent $z$. Section III's scaling prediction, $z=d$, thus yields explicit numerical values for $x$ and $y$ in any dimension $d$.

Also of interest is the behavior of the specific heat, $C_{P}$, near the onset of superfluidity and at low $T$ in both the Bose glass and superfluid states. Using (5.1) and the definition $C_{P}=-T \partial^{2} f / \partial T^{2}$, one obtains

$$
C_{P} \sim T^{d / z}
$$

at $\rho=\rho_{c}$. In the superfluid phase, the lowest-lying excitations are the fourth sound modes discussed in Sec. III. The energy, $\omega$, of the mode with wave vector $k$ is [see (3.13) and (5.5)] $\omega=C_{2} k$ for small $k$, implying an energy density proportional to $T^{d+1} / C_{2}^{d}$ in $d$ dimensions, or a specific heat which behaves [see (5.5)] like

$$
C_{P} \sim\left(T / C_{2}\right)^{d} \sim T^{d} / \delta^{v d(z-1)} \sim T\left(T / T_{c}\right)^{d-1}
$$

for $T \ll T_{c}$. The existence of a finite, nonzero density of states for particle and hole excitations of arbitrarily low energy in the Bose glass phase, by contrast, gives rise at low $T$ to the linear specific heat, $C_{P} \sim T$, typical of disordered systems. ${ }^{21}$

To obtain detailed experimental predictions from the relations derived in this section requires knowledge of the exponents $v$ and $z$ appropriate to the experiment. Consider, for example, ${ }^{4} \mathrm{He}$ in porous media such as Vycor ${ }^{15}$ or Silica Gel, ${ }^{16}$ which consist of random threedimensional (3D) networks of interconnected pores or channels of diameter roughly $20-70 \AA$; the pore size can be varied from sample to sample. The onset of superfluidity occurs at critical densities, $\rho_{c}$, roughly corresponding to two monolayers of ${ }^{4} \mathrm{He}$ deposited on the inside walls of the pores. Previous theories ${ }^{6}$ have modelled the superfluid onset by assuming that for $\rho<\rho_{c}$ the ${ }^{4} \mathrm{He}$ atoms simply adhere to the walls, preferentially "filling up" the lowest potential wells produced by the random wall surfaces. At $\rho=\rho_{c}$ the pore walls are assumed completely plated by an inert covering of bound ${ }^{4} \mathrm{He}$ atoms, which smooth out the effective wall potential experienced by further ${ }^{4} \mathrm{He}$ atoms introduced as $\rho$ increases beyond $\rho_{c}$. These extra atoms were assumed to move in an essentially uniform static potential, and so are treated as a dilute Bose gas, free of any external potential. Thus at $T=0$ they Bose condense immediately, as $\rho$ increases above $\rho_{c}$, thus producing, at $\rho=\rho_{c}$, the ideal or generic mean-field superfluid transition appropriate to pure systems, as discussed in Sec. IV A. In this scenario, $v=\frac{1}{2}$ and $z=2$, producing the results

$$
T_{c} \sim\left[\rho_{s}(0)\right]^{x}, \quad T_{c} \sim\left[C_{2}(0)\right]^{y},
$$

and $\rho_{s}(0) \sim\left(\rho-\rho_{c}\right)^{5}$, with [see (5.8)-(5.10)]

$$
x=2 / d ; y=2 ; \xi=1 \text {. }
$$

Since $d_{c}=2$ for the ideal transition, the values in (5.13) derived from scaling are not correct for $d>2$. (For $d<2$, $x$ and $y$ are actually ill defined since $T_{c} \equiv 0$ ). It turns out that for $d>2, x$ and $\zeta$ are as given in (5.13) but $y=4 / d$.

We view this approximation of the perfect screening of the random wall potential by an inert deposited ${ }^{4} \mathrm{He}$ layer as somewhat unrealistic. It is clear that there will be a finite rate of exchange among ${ }^{4} \mathrm{He}$ atoms bound to the wall at different positions, and between atoms bound to the wall and those relatively free to wander over it. Moreover, even the relatively free atoms feel some nonzero random potential. Thus it seems more likely that the superfluid onset transition belongs in the universality class of the Bose glass-superfluid transition discussed in Secs. II, IV B, and IV C. Under this assumption we can invoke the results of Sec. III, $z=d=3$ and $v \geq 2 / d=\frac{2}{3}$, whereupon $[(5.8)-(5.10)]$

$$
\begin{aligned}
& x=\frac{3}{4} ; \\
& y=\frac{3}{2} ; \\
& \zeta=4 v \geq \frac{8}{3} .
\end{aligned}
$$

Other predictions for measurable quantities follow similarly.

While existing data do not allow the unambiguous elimination of either (5.13) or (5.14), there is room for optimism that further experiments will distinguish between the two sets of predictions, which differ significantly. For ${ }^{4} \mathrm{He}$ in Vycor there is evidence for an exponent $x$ slightly 
larger than the ideal $\frac{2}{3}$, and more consistent with $\frac{3}{4}$, as well as a tail in $\rho_{s}(0)$ as $\rho$ is decreased, ${ }^{15}$ suggestive of a crossover from a linear region (with the ideal exponent $\zeta=1)$ to a nontrivial onset critical region with an exponent ${ }^{40} \xi$ of order 2 . Such a narrow critical region for zero-temperature onset would be expected if the rate of exchange with the atoms in the layers closest to the walls is small. A wider and more accessible quantum critical region is expected in systems with strong disorder, uncorrelated down to the atomic scale, in which the exchange is large. Preliminary fits to the data on ${ }^{4} \mathrm{He}$ in carbon black, ${ }^{17}$ which exhibits a wider apparent critical region for $\rho_{s}(0)$ as a function of $\rho$, yield a value of $\zeta \approx 2.5$ and $x \approx 0.8$.

Further possible difficulties of interpretation might result from the very weak irrelevance of disorder at the finite-temperature transition to superfluidity; the crossover exponent, $\alpha_{I}$ (simply the specific-heat exponent of the 3D $X Y$ model), is negative but extremely small. Thus the crossover from $T=0$ to finite-temperature behavior may be complicated by the simultaneous slow disappearance of the effects of disorder. The measured exponent, $v_{T}$, as inferred from the superfluid density and associated with the superfluid transition at low but nonzero temperature in Silica $\mathrm{Gel}^{41}$ is roughly 1 , considerably larger than the expected 3D $X Y$-model value of $\sim \frac{2}{3}$, but perhaps consistent with transients associated with the weakly irrelevant disorder.

Similar remarks apply to the ${ }^{4} \mathrm{He}$ films on 2D substrate systems. Again, there is a significant difference between the predictions of the inert layer picture $\left(v=\frac{1}{2}, z=d=2\right)$ and our Bose glass-superfluid predictions $(z=d=2$, $v \geq 1$ ) for some measurable quantities, notably the exponent $\zeta$ of the zero-temperature superfluid density (5.10). [In $d=2$ the scaling results (5.8)-(5.10) apply even to the inert layer model, aside from possible logarithmic corrections.] It is clear that further experiments designed to distinguish between the two sets of predictions would be extremely valuable.

We now briefly summarize our main conclusions. For bosons hopping on a lattice with short-range repulsive interactions and disorder, we find three possible groundstate phases: (1) an incompressible Mott insulator with a density of bosons commensurate with the lattice and a gap for particle and hole excitations; (2) a gapless Bose glass insulator with finite compressibility, exponentially decaying superfluid correlations in space, yet an infinite superfluid susceptibility; (3) a superfluid phase with the usual off-diagonal long-ranged order.

In the absence of disorder only the Mott and superfluid phases are present. Contrary to naive expectations, the zero-temperature transition to superfluidity from the Mott phase is generically not in the universality class of the $d+1$-dimensional $X Y$ model. Such $X Y$ transitions occur only at special multicritical points (see Fig. 1). Generically the transition is an ideal transition, like the zero-density transition which occurs as the density of bosons is increased from zero in the absence of an external potential. Similar conclusions apply to quantum $X Y$ magnets in an imposed field in the $z$ direction, which breaks the time-reversal invariance.
When disorder is present, the gapless Bose glass phase intervenes between the Mott and superfluid phases, so that the zero-temperature superfluid onset transition always occurs from the Bose glass [as in Fig. 2(a)]. The critical properties of this transition are characterized by three exponents: A dynamic exponent $z$ which we argue is equal to $d$, a correlation length exponent which satisfies the bound $v \geq 2 / d$, and an order-parameter exponent which should satisfy the bound, $\eta \leq 2-d$. These exponent relations, which are verified by direct calculation in $d=1\left(z=1, v=\infty\right.$ and $\left.\eta=\frac{2}{3}\right)$, allow stringent bounds to be placed on experimentally measurable exponents for ${ }^{4} \mathrm{He}$ absorbed in porous media or on disordered substrates. More surprisingly, several measurable exponents, depending only on $z$, can be predicted exactly.

We argue that if a simple high-dimensional limit exists at all for the Bose glass to superfluid transition, it cannot be of the conventional Gaussian or mean-field kind. This leads us to speculate that the upper critical dimension may in fact be infinite, with, for example, the equality $z=d$ holding in all dimensions. If this is indeed the case, other microscopic approaches to calculate exponents explicitly away from $d=1$ are clearly highly desirable. It is plausible that a $1+\varepsilon$ expansion could be developed, although at first sight at least this appears more difficult than studying the classical $X Y$ model in $2+\varepsilon$ dimensions, which at present we do not know how to do. Other approaches, perhaps exploiting the generalization ${ }^{4}$ of Haldane's representation ${ }^{13}$ to $d>1$, might conceivably be more fruitful.

\section{ACKNOWLEDGMENTS}

We are very grateful to K. Blum, M. C. Cross, S. M. Girvin, B. I. Halperin, D. H. Lee, P. A. Lee, J. Reppy, D. Rokhsar, M. Schick, and J. Toner for useful discussions. Thanks are also due to the Aspen Center for Physics where this work was begun. P.B.W. gratefully acknowledges financial support from the Weingart Foundation through Caltech and the National Science Foundation (NSF) through Grant No. DMR8412543. D.S.F. gratefully acknowleges the support of the A. P. Sloan Foundation and the NSF under Grant No. DMR8719523.

\section{APPENDIX A: PHASE DIAGRAM IN MEAN-FIELD THEORY}

In this Appendix we study the Hamiltonian (2.1) in the limit of infinite-range hopping. In particular, we obtain the exact zero-temperature phase diagram both in the pure case and with on-site disorder.

In the absence of hopping, $J_{i j}=0$, the Hamiltonian in (2.1) reduces to a sum of on-site terms and can then be easily diagonalized. This fact suggests that progress can be made by factorizing the hopping term (see below). To this end, it is convenient to express the partition function, $Z=\operatorname{Tr} \exp (-\beta H)$, in the interaction representation as

$$
\begin{aligned}
Z & =\operatorname{Tr} \exp \left(-\beta H_{0}\right)\left\{T_{\tau} \exp \left[-\frac{1}{\hbar} \int_{0}^{\beta \hbar} d \tau H_{1}(\tau)\right]\right\} \\
& =Z_{0}\left\langle T_{\tau} \exp \left[-\frac{1}{\hbar} \int_{0}^{\beta \hbar} d \tau H_{1}(\tau)\right]\right\rangle_{0}
\end{aligned}
$$


with $Z_{0}=\operatorname{Tr} \exp \left(-\beta H_{0}\right)$,

$$
H_{1}(\tau)=e^{\tau H_{0}} H_{1} e^{-\tau H_{0}},
$$

and the average taken in the ensemble given by $H_{0}$. Here $T_{\tau}$ is the imaginary time ordering operator. Note that this is the opposite of the standard many-body perturbation theoretic approach; ${ }^{19}$ i.e., the roles of $H_{0}$ and $H_{1}$ are essentially reversed. The partition function $Z_{0}$ is simply a product of on-site partition functions and can be easily computed. A complex $c$-number field $\psi_{i}(\tau)$ can now be introduced ${ }^{9,10}$ to factorize (or decouple) the off-site hopping term $H_{1}$, enabling the partition function to be expressed as

$$
Z=Z_{0} \int \prod_{i} D \psi_{i}(\tau) D \psi_{i}^{*}(\tau) \exp \left[-S\left(\psi_{i}\right)\right]
$$

with an effective action

$$
\begin{aligned}
S\left(\psi_{i}\right)= & \sum_{i, j} \int d \tau\left(J^{-1}\right)_{i j} \psi_{i}^{*}(\tau) \psi_{j}(\tau) \\
& -\sum_{i} \ln \left\langle T_{\tau} \exp \left[\int d \tau \psi_{i}(\tau) \hat{\boldsymbol{\Phi}}_{i}^{+}(\tau)+\text { H.c. }\right]\right\rangle_{0},
\end{aligned}
$$

where $\left(J^{-1}\right)_{i j}$ is the inverse of the matrix of hopping rates. The average in (A4), which is now on-site in the original boson field $\Phi_{i}$, can be readily computed as a cumulant expansion in powers of $\psi_{i}$. Since $\left\langle\psi_{i}\right\rangle$ is linearly related to $\left\langle\Phi_{i}\right\rangle$, the field $\psi_{i}$ serves as an order parameter for superfluidity.

Equations (A3) and (A4) represent an exact rewriting of the original partition function and thereby serves as a convenient starting point to generate a field theoretic description of the onset of superfluidity (see Sec. IV A). Here, we pursue a different path, specializing to the infinite-range hopping limit of (2.1), namely $J_{i j} \equiv J / N$, where $N$ is the total number of lattice sites in the system. In this limit $H_{1}$ can be expressed as a perfect square,

$$
H_{1}=-(J / N)\left[\sum_{i} \hat{\boldsymbol{\Phi}}_{i}^{+}\right]\left[\sum_{i} \hat{\boldsymbol{\Phi}}_{i}\right]
$$

and only a single auxiliary field $\psi(\tau)$ is needed to decouple the nonlocal hopping term. The action in (A4) then takes the form $S_{\infty}(\psi)=N f(\psi)$, so that in the thermodynamic limit a saddle point evaluation of the functional integral in (A3) becomes exact. With the assumption that the lowest-energy saddle point solution involves a time independent field, $\psi(\tau)=\psi$, one finds upon performing the cumulant expansion in (A4) an action of the typical Landau form

$S_{\infty}(\psi)=\beta N\left[\frac{1}{2} r(\mu, J, T)|\psi|^{2}+u(\mu, T)|\psi|^{4}+O\left(|\psi|^{6}\right)\right]$.

At $T=0, r(\mu, J)$ is given explicitly by

$$
r(\mu, J)=(2 J)^{-1}-\left(\frac{1}{2} N\right) \sum_{i} A_{i}(\mu, J),
$$

with

$$
A_{i}=\frac{V\left(n_{i}+1\right)}{V n_{i}-\left(J+\mu+\delta \mu_{i}\right)}+\frac{V n_{i}}{\left(J+\mu+\delta \mu_{i}\right)-V\left(n_{i}-1\right)} .
$$

Here, $n_{i}$, the smallest non-negative integer larger than $\left(J+\mu+\delta \mu_{i}\right) / V$, is the number of bosons which minimizes the energy on the $i$ th site in the absence of hopping $\left(H_{1}=0\right)$. Moreover, the denominators in (A7b) correspond to the energy required (at $H_{1}=0$ ) to change the number of bosons on the $i$ th site from $n_{i}$ to $n_{i} \pm 1$.

Minimization of the action $S_{\infty}(\psi)$ indicates that the phase boundary between the superfluid $(\langle\psi\rangle=0)$ and normal $(\langle\psi\rangle \neq 0)$ states corresponds to the condition $r(\mu, J)=0$. This enables an explicit construction of the $T=0$ phase diagram. The phase diagram for the pure case $\left(\delta \mu_{i}=0\right)$ is shown in Fig. 1. Notice the lobes of normal phase. In the presence of on-site disorder with a smooth distribution for $\delta \mu_{i}$, as in (2.2), the sum in (A7a) can be converted into an integral (in the thermodynamic limit). An explicit phase diagram is shown in Fig. 4 for a uniform distribution between $-\Delta$ and $\Delta$, with $\Delta<V / 2$. Note that the normal phase lobes have shrunk in the presence of disorder. Indeed, for $\Delta>V / 2$ or for an unbounded distribution as in $(2.2 b)$ the normal phase disappears entirely (except, of course, at $J=0$ ). This is due to the singularities in the sum (or integral) in (A7a), at values of $\delta \mu$ which correspond to vanishing energy denominators in (A7b). Physically, sites with these special values of $\delta \mu$ have a degenerate energy (at $H_{1}=0$ ) for $n$ and $n \pm 1$ bosons, and can thus form a superposition with a nonzero on-site (superfluid) order parameter. In the presence of an (arbitrarily small) infinite-range hopping term, these special sites serve as seeds for global superfluid onset, as emphasized by MHL. ${ }^{4}$

From the above exact solution of the infinite-range hopping model it is also possible to extract the average boson density per site $\left\langle N_{i}\right\rangle$, using the identity

$$
\left\langle\hat{\mathbf{N}}_{i}\right\rangle=\frac{1}{\beta N} \frac{\partial}{\partial \mu} \ln Z \text {. }
$$

In the normal phase $(\langle\psi\rangle=0)$ the action at the saddle point vanishes, so that the only contribution to the density comes from the (trivial) on-site partition function, $Z_{0}$. For the pure case one finds $\left\langle N_{i}\right\rangle=n$ for $n-1<\mu / V<n$. The normal regions in the phase diagram in Fig. 1, thus correspond to locked commensurate phases with an integer number of bosons per site. As discussed in Sec. II, these phases are in fact Mott insulators. In the presence of weak bounded disorder $(\Delta<V / 2)$ one likewise deduces that $\left\langle N_{i}\right\rangle$ is locked to an integer $n$, in each of the insulating lobes (see Fig. 4).

\section{APPENDIX B: BOSONS IN ONE DIMENSION}

In this Appendix we review Haldane's 13 “densityrepresentation" for one-dimensional boson systems and following Giamarchi and Schulz ${ }^{5}$ obtain a functional integral representation for the $T=0$ partition function of bosons moving in a periodic plus random potential. This functional integral representation is employed in Sec. 
IV B to study the critical behavior of the zerotemperature superfluid onset transition in one dimension.

Following Haldane, ${ }^{13}$ we express the boson field operator $\Phi$ in terms of the density $\rho(x)$ and phase $\phi(x)$ as $\Phi(x)=\sqrt{\rho(x)} \exp [i \phi(x)]$. The density operator is written as

$$
\rho(x)=\left[\rho_{0}+\Pi(x)\right] \sum_{m=-\infty}^{\infty} e^{i 2 m \theta(x)},
$$

where $\rho_{0}$ is the mean density and $\Pi(x)$ a small deviation which satisfies the canonical commutation relations

$$
\left[\phi(x), \Pi\left(x^{\prime}\right)\right]=i \delta\left(x-x^{\prime}\right) .
$$

The term multiplying $\rho_{0}+\Pi$ in (B1) accounts for the Bose particles' discreteness, the correct treatment of which is crucial for insuring the existence of both the Mott insulating and Bose glass states. Specifically, the field $\theta(x)$ is defined via

$$
\partial_{x} \theta(x)=\pi\left[\rho_{0}+\Pi(x)\right],
$$

the position of the Bose particles therefore being taken as the points where $\theta(x)=n \pi$, for integer $n$.

The Hamiltonian describing the long-wavelength and low-energy excitations of the superfluid state can be written [cf. (3.13)],

$$
H_{0}=\frac{1}{2} \int d x\left[\kappa^{-1} \Pi^{2}+\left(\rho_{s} / m\right)\left(\partial_{x} \phi\right)^{2}\right],
$$

with $\hbar=1$. For a Galilean invariant system at $T=0, \rho_{s}$ equals $\rho_{0}$, the total density, but will be renormalized in the presence of external potentials. The algebraicallydecaying correlations of the superfluid state follow from (B4), since

$$
\left\langle\hat{\boldsymbol{\Phi}}^{+}(x) \hat{\boldsymbol{\Phi}}(0)\right\rangle \sim|x|^{-K / 2},
$$

with

$$
(\pi K)^{2} \equiv m / \rho_{s} \kappa
$$

It will sometimes be convenient to express results in terms of $K$ and the (second) sound speed $C_{2}=\left(\rho_{s}\right)$ $m \kappa)^{1 / 2}$, rather than $\rho_{s}$ and $\kappa$.

Periodic and random potentials are introduced via extra terms in the Hamiltonian: $H=H_{0}+H_{P}+H_{R}$, with

$$
\begin{aligned}
& H_{P}=-u \int d x \cos (2 \pi x) \rho(x), \\
& H_{R}=\int d x V(x) \rho(x),
\end{aligned}
$$

with $V(x)$ a random potential to be specified below. In the following analysis we shall consider only densities near or at the value $\rho_{0}=1$, which is commensurate with the periodic potential.

As in Secs. II and III, the phase diagram and associated transitions can be studied most conveniently by expressing the partition function in a path integral representation. Since $H_{P}$ and $H_{R}$ involve the density, rather than the phase, $\phi$, of the order parameter, the partition function is most easily represented as a functional integral in a basis of states that diagonalizes the operator $\widetilde{\theta}(x)$, which is directly related to the density fluctuations

$$
\partial_{x} \widetilde{\theta}(x) \equiv \pi \Pi(x) \text {. }
$$

Since $\widetilde{\theta}$ satisfies the commutation relations,

$$
\left[\widetilde{\theta}(x), \pi^{-1} \partial_{x} \phi\left(x^{\prime}\right)\right]=i \delta\left(x-x^{\prime}\right),
$$

$H_{0}$ can also be expressed in terms of $\widetilde{\theta}$, whereupon the partition function at $T=0$ can be written

$$
Z=\int D \widetilde{\theta}(x, \tau) \exp (-S) \text {. }
$$

The action $S$ equals $S_{0}+S_{P}+S_{R}$, where

$$
S_{0}=\frac{K}{2 \pi} \int d x d \tau\left[C_{2}^{-1}\left(\partial_{\tau} \widetilde{\theta}\right)^{2}+C_{2}\left(\partial_{x} \widetilde{\theta}\right)^{2}\right] \text {. }
$$

The action associated with $H_{P}$ and $H_{R}$ can be obtained by inserting (B1) into (B6) and retaining only the most important terms. Since for weak external potentials $\widetilde{\theta}(x, \tau)$ is slowly varying, one need only keep the $m= \pm 1$ contributions to $H_{P}\left(\right.$ since $\left.\rho_{0} \simeq 1\right)$ and the $m=0, \pm 1$ contributions to $H_{R}$. One thereby obtains

$$
\begin{aligned}
& S_{P}=-u \int_{x, \tau} \cos \left[2 \pi\left(\rho_{0}-1\right) x+2 \widetilde{\theta}(x, \tau)\right], \\
& S_{R}=-\int_{x, \tau} \delta \mu(x) \partial_{x} \widetilde{\theta}(x, \tau)+\int_{x, \tau}\left[\xi(x) e^{i 2 \tilde{\theta}(x, \tau)}+\text { c.c. }\right],
\end{aligned}
$$

where $-\delta \mu(x)$ and $\xi(x)$ are the contributions from $V(x)$ with Fourier components near $k=0$ and $k= \pm 2 \pi \rho_{0}$, respectively. The potential $\xi(x)$ is thus complex and will be taken to satisfy a (complex) Gaussian white noise distribution,

$$
\overline{\xi(x)}=0, \quad \overline{\xi^{*}(x) \xi\left(x^{\prime}\right)}=D \delta\left(x-x^{\prime}\right) .
$$

The real random chemical potential field $\delta \mu(x)$ will also be taken as Gaussian:

$$
\overline{\delta \mu(x)}=0, \quad \overline{\delta \mu(x) \delta \mu\left(x^{\prime}\right)}=\Delta \delta\left(x-x^{\prime}\right) .
$$

${ }^{1}$ See P. A. Lee and T. V. Ramakrishnan, Rev. Mod. Phys. 57, 287 (1985), and references therein.

${ }^{2}$ See A. J. Leggett, Physica Fennica 8, 125 (1973), for an argument that no Bose system can be a normal (nonsuperfluid) conducting fluid at $T=0$.

${ }^{3}$ D. S. Fisher and M. P. A. Fisher, Phys. Rev. Lett. 61, 1847 (1988).

${ }^{4}$ M. Ma, B. I. Halperin, and P. A. Lee, Phys. Rev. B 34, 3136 (1986).
${ }^{5}$ T. Giamarchi and H. J. Schulz, Europhys. Lett. 3, 1287 (1987); Phys. Rev. B 37, 325 (1988).

${ }^{6}$ P. B. Weichman, M. Rasolt, M. E. Fisher, and M. J. Stephen, Phys. Rev. B 33, 4632 (1986).

${ }^{7}$ D. I. Uzunov, Phys. Lett. 87A, 11 (1981).

${ }^{8}$ See P. B. Weichman, Phys. Rev. B 38, 8739 (1988); P. B. Weichman and K. Kim (unpublished).

${ }^{9}$ S. Doniach, Phys. Rev. B 24, 5063 (1981).

${ }^{10}$ M. P. A. Fisher and G. Grinstein, Phys. Rev. Lett. 60, 208 
(1988).

${ }^{11}$ M. E. Fisher, M. N. Barber, and D. Jasnow, Phys. Rev. A 8, 1111 (1973).

12 J. T. Chayes, L. Chayes, D. S. Fisher, and T. Spencer, Phys. Rev. Lett. 57, 2999 (1986); Commun. Math. Phys. (to be published).

${ }^{13}$ F. D. M. Haldane, Phys. Rev. Lett. 47, 1840 (1981).

${ }^{14}$ M. P. A. Fisher and D. H. Lee, Phys. Rev. B 39, 2756 (1989).

15J. D. Reppy, Physica 126B, 335 (1984).

${ }^{16}$ D. Finotello, K. A. Gillis, A. Wong, and M. H. W. Chan, Phys. Rev. Lett. 61, 1954 (1988).

${ }^{17}$ K. I. Blum, J. S. Souris, and J. D. Reppy (unpublished).

${ }^{18}$ M. E. Fisher, Rep. Prog. Phys. 30, 615 (1967), and references therein.

${ }^{19}$ See, e.g., A. L. Fetter and J. D. Walecka, Quantum Theory of Many Particle Systems (McGraw-Hill, New York, 1971), for a discussion of the necessary formalism.

${ }^{20} \mathrm{H}$. Von Dreifus and A. Klein (unpublished).

${ }^{21}$ For a discussion of analogous properties in a Fermi glass, see R. Freedman and J. A. Hertz, Phys. Rev. B 15, 2384 (1977).

${ }^{22}$ R. B. Griffiths, Phys. Rev. Lett. 23, 17 (1969).

${ }^{23}$ B. M. McCoy and T. T. Wu, Phys. Rev. 176, 631 (1968); B. M. McCoy, Phys. Rev. B 18, 1014 (1969).

${ }^{24}$ P. C. Hohenberg and B. I. Halperin, Rev. Mod. Phys. 49, 435 (1977).

${ }^{25}$ See, e.g., A. Casher, D. Lurié, and M. Revzen, J. Math. Phys. 9, 1312 (1968).

${ }^{26}$ See, e.g., M. E. Fisher, in Critical Phenomena, Vol. 186 of Lecture Notes in Physics, edited by F. J. W. Hahne (Springer, Berlin, 1983), p. 1.

${ }^{27}$ This definition is chosen so that the usual scaling law, $\gamma=(2-\eta) v$, holds, where $\gamma$ describes the divergence of the static susceptibility $\bar{\chi}(k=0, \omega=0)$.

${ }^{28}$ V. N. Popov, Functional Integrals in Quantum Field Theory and Statistical Physics (Riedel, Dordrecht, 1983), Chap. 6. The form of the effective action, (3.13), can be obtained from Popov's results by integrating out the amplitude fluctuations, $\pi$, to quadratic order.

${ }^{29}$ See Ref. 11 for a detailed discussion of the finite-size scaling approach to the superfluid density.

${ }^{30}$ Similar arguments fix the dynamical exponent at the finitetemperature $\lambda$ transition in ${ }^{4} \mathrm{He}$. See Ref. 24.

${ }^{31}$ The proof given in Ref. 12 applies for $(d+1)$-dimensional quantum systems with the randomness depending on only the $d$ spatial dimensions.

${ }^{32}$ T. Ohta and D. Jasnow, Phys. Rev. B 20, 139 (1979).

33J. M. Kosterlitz and D. J. Thouless, J. Phys. C 6, 1181 (1973); J. M. Kosterlitz, J. Phys. C 71, 1046 (1974).

${ }^{34}$ S. T. Chui and J. D. Weeks, Phys. Rev. B 14, 4978 (1976).

${ }^{35}$ S. N. Dorogovstev, Phys. Lett. 76A, 169 (1980); D. Boyanovsky and J. L. Cardy, Phys. Rev. B 26, 154 (1982); I. D. Laurie and V. V. Prudnikov, J. Phys. C 17, 1655 (1984).

${ }^{36} \mathrm{P}$. B. Weichman and K. Kim (unpublished).

${ }^{37}$ See B. Stolan, E. Pytte, and G. Grinstein, Phys. Rev. B 30, 1506 (1984).

${ }^{38}$ D. McQuenney, G. Agnolet, and J. D. Reppy, Phys. Rev. Lett. 52, 1325 (1984).

${ }^{39}$ See, e.g., M. Ma, Modern Theory of Critical Phenomena (Benjamin, Reading, 1976).

${ }^{40} \mathrm{~K}$. Blum, private communication.

${ }^{41}$ M. H. W. Chan, K. I. Blum, S. Q. Murphy, G. K. S. Wong, and J. D. Reppy, Phys. Rev. Lett. 61, 1950 (1988). 